\title{
Un análisis de revisiones de modelos y algoritmos para la optimización de planes de aprovisionamiento, producción y distribución de la cadena de suministro
}

\author{
Guzmán E , Poler R , Andres B
}

Recibido: 31 de Mayo de 2019

https://doi.org/10.37610/dyo.v0i70.567

Aceptado: 17 de Enero de 2020

\section{Resumen}

El propósito de este artículo es analizar los diferentes modelos y algoritmos utilizados para la optimización de planes de aprovisionamiento, producción y distribución en la cadena de suministro a través de las últimas revisiones de la literatura realizadas en este campo. Este estudio pretende proporcionar a los investigadores un punto de partida para la selección de modelos y algoritmos para la resolución de este tipo de problemas en la cadena de suministro y presentar varias líneas de investigación futuras.

\section{Palabras clave}

Cadena de suministro, planificación, optimización, modelos, algoritmos, revisión de la literatura

\section{Introducción}

Desde que en 1982, Keith Oliver, un consultor de Boaz Allen Hamilton, introdujo el término "gestión de la cadena de suministro" en una entrevista para el Financial Times (Handfield y Nichols 1999), diferentes modelos y métodos para el diseño y la gestión de la cadena de suministro se han publicado en diversos estudios que, generalmente, son de tipo principal (investigación original), mientras que otros son de tipo secundarios (revisiones de la literatura), siendo escasos los estudios terciarios (análisis de revisiones de la literatura). Kazemi et al. (2018), en su artículo de revisión de la cadena de suministro de ciclo cerrado, comentan que, por el volumen de publicaciones que aparecen constantemente y su crecimiento en los últimos años, es difícil mantener una visión amplia del área de estudio, siendo necesaria la contribución de estudios secundarios o terciarios que puedan ayudar a sintetizar y estructurar cada área de investigación.

El presente artículo proporciona un estudio terciario, realizando un análisis sistemático de las revisiones de la literatura sobre modelos y algoritmos para la optimización de planes

\footnotetext{
Eduardo Guzmán *

eguzman@cigip.upv.es

https://orcid.org/0000-0003-0866-2095

Raul Poler *

rpoler@cigip.upv.es

https://orcid.org/0000-0003-4475-6371

Beatriz Andrés *

bandres@cigip.upv.es

https://orcid.org/0000-0002-7920-7711

* Centro de Investigación en Gestión e Ingeniería de la

Producción (CIGIP) Escuela Politécnica Superior de Alcoy,

Universitat Politècnica de València (UPV). Calle Alarcón,

03801 Alcoy (España).
}

de aprovisionamiento, producción y distribución en la cadena de suministro y propone directrices útiles para la investigación y el desarrollo de nuevos modelos y algoritmos en dichas áreas. Para la realización de este estudio se establecieron las siguientes preguntas de investigación:

PI1: ¿Cuáles son las perspectivas o enfoques que se proponen en la literatura respecto a la cadena de suministro?

PI2: ¿Cuál es el estado actual de las investigaciones realizadas en la optimización de planes de aprovisionamiento, producción y distribución?

PI3: ¿Cuáles son las revisiones más relevantes en el contexto de optimización de planes de aprovisionamiento producción y distribución en la cadena de suministro?

PI4: ¿Como conceptualizar los planes de aprovisionamiento, producción y distribución?

PI5: ¿Como conceptualizar los métodos cuantitativos usados en la optimización de los planes de aprovisionamiento, producción y distribución?

PI6: ¿Que métodos cuantitativos se han usado en la literatura para los planes de aprovisionamiento, producción y distribución?

PI7: ¿Que líneas de investigación futura se pueden plantear en base a las revisiones existentes y que áreas de investigación se pueden orientar para futuros estudios?

Para responder a estas preguntas de investigación, se ha realizado un análisis sistemático de las revisiones de la literatura centradas en los problemas de planificación de la cadena de suministro. Se evaluaron un total 168 artículos de los que se seleccionaron 17 para realizar un análisis en profundidad. El criterio de selección se basó, principalmente, en 
revisiones que hubieran analizado modelos matemáticos o métodos cuantitativos y que hubieran utilizado un proceso metodológico en la revisión. De forma general, las investigaciones encontradas plantean enfoques de revisión como el sistemático, narrativo y meta-análisis, sin embargo, este estudio se enfoca en las revisiones sistemáticas para facilitar la comparación de los métodos cuantitativos utilizados en los diferentes enfoques de la cadena de suministro. Además, se utiliza el modelo SCOR para la clasificación de los análisis elaborados por los artículos seleccionados.

Este análisis pretende proporcionar un resumen conciso del tipo de planificación abordada en los artículos de revisión, así como los modelos y métodos de resolución de problemas de optimización, los algoritmos de resolución y los enfoques. Además, con esta investigación se pretende identificar brechas y oportunidades para proponer líneas de investigación futura en el ámbito de la planificación de operaciones en la cadena de suministro.

Este articulo está organizado de la siguiente forma: la sección 1 muestra los enfoques o perspectivas estudiadas en la literatura y la definición de éstos. La sección 2 explica la metodología utilizada en el análisis sistemático. La sección 3 presenta los hallazgos de la investigación. La sección 4 muestra las observaciones, las perspectivas y las orientaciones que dan los investigadores. Por último, la sección 5 presenta las conclusiones y las orientaciones para futuras investigaciones.

\section{Perspectivas de las revisiones}

El concepto de cadena de suministro ha sido descrito por varios autores, los cuales expresan rasgos similares en sus definiciones. A su vez, estas definiciones se adaptan al análisis o al ámbito de investigación. Una definición que resume de forma apropiada este término es la que proporcionan Ivanov y Sokolov (2010): "una cadena de suministro es una red de organizaciones, flujos y procesos en la que varias empresas (proveedores, fabricantes, distribuidores y minoristas) colaboran (cooperan y coordinan) a lo largo de toda la cadena de valor para adquirir materias primas, para convertir estas materias primas materiales en productos finales especificados para entregar estos productos finales a los clientes".

Es importante resaltar los enfoques o perspectivas de la cadena de suministro y la orientación que están dando actualmente los investigadores, por lo que la Tabla 1 describe los ámbitos de investigación de la cadena de suministro, que se encuentran en la literatura. Las definiciones de estos términos pretenden dar respuesta a la PI1. 
Tabla 1 Perspectivas de análisis de la cadena de suministro

\begin{tabular}{ll}
\hline Perspectiva & Definición \\
\hline $\begin{array}{l}\text { Gestión de la cadena de } \\
\text { suministro (Supply Chain }\end{array}$ & $\begin{array}{l}\text { La gestión de la cadena de suministro es la gestión de los flujos de materiales, información y financieros } \\
\text { a través de la cadena de suministro. Incluye la coordinación y colaboración de procesos y actividades } \\
\text { Management) }\end{array}$ \\
$\begin{array}{l}\text { a través de diferentes funciones tales como marketing, ventas, producción, diseño de productos, adqui- } \\
\text { siciones, logística, finanzas y tecnología de la información dentro de la cadena de suministro (Sodhi } \\
\text { y Tang 2012). }\end{array}$ \\
\hline
\end{tabular}

Cadena de suministro bajo incertidumbre (Supply Chain under Uncertainty)

Las cadenas de suministro que tratan el tema de la incertidumbre expresan que la incertidumbre se propaga hacia arriba y hacia abajo influyendo en su desempeño. La incertidumbre afecta a los volúmenes de demanda y las capacidades de oferta que están sujetos a la inestabilidad económica y las fluctuaciones del mercado, además de otros factores endógenos y exógenos (Fathian et al. 2018).

Gestión de la cadena de suministro de ciclo cerra-

Una cadena de suministro de ciclo cerrado puede verse como la tradicional cadena de suministro complementada con operaciones inversas para los productos recuperados que se reprocesan y finalmente vuelven a entrar en la cadena de suministro. Mientras que la gestión de la cadena de suministro de ciclo cerrado describe el diseño, control y operación de un sistema para maximizar la creación de valor a lo largo de todo el ciclo de vida de un producto, con la recuperación dinámica de valor de diferentes tipos y volúmenes de rendimientos a lo largo del tiempo (Stindt y Sahamie 2014).

Chain Management)

\begin{tabular}{ll}
$\begin{array}{l}\text { Gestión sostenible de } \\
\text { la cadena de suministro } \\
\text { (Sustainable Supply Chain } \\
\text { Management) }\end{array}$ & $\begin{array}{l}\text { La gestión sostenible de la cadena de suministro es una extensión del concepto tradicional de gestión } \\
\text { de ladena de suministro que agrega aspectos económicos, ambientales y sociales / éticos, tomando } \\
\text { en cuenta los exigencias de los clientes y los demás stakeholders (grupos de interés) (Sacaluga et al. } \\
\text { 2011; Patel y Desai 2018) }\end{array}$ \\
\hline $\begin{array}{l}\text { Gestión del riesgo de } \\
\text { la cadena de suministro } \\
\text { (Supply Chain Risk Man- } \\
\text { agement) }\end{array}$ & $\begin{array}{l}\text { La gestión del riesgo de la cadena de suministro es un área multidisciplinaria con investigación y prác- } \\
\text { tica que se basa en al menos tres campos: la gestión de la cadena de suministro, la gestión del riesgo } \\
\text { empresarial (identificación, evaluación, mitigación, respuesta), y la gestión de crisis (Sodhi y Tang } \\
\text { 2012; Fahimnia et al. 2015). }\end{array}$ \\
\hline $\begin{array}{l}\text { Gestión de la cadena de } \\
\text { suministro verde (Green }\end{array}$ & $\begin{array}{l}\text { Es la integración del pensamiento ambiental en la gestión de la cadena de suministro, incluido el diseño } \\
\text { del producto, la selección y abastecimiento de materiales, los procesos de fabricación, la entrega del } \\
\text { producto final a los consumidores, así como la gestión al final de la vida útil del producto (Srivastava } \\
\text { ment) Chain Manage- }\end{array}$ \\
\hline $\begin{array}{l}\text { 2007). } \\
\text { Resiliencia de la cadena } \\
\text { de suministro (Supply }\end{array}$ & $\begin{array}{l}\text { Trata de la capacidad de adaptación de una cadena de suministro para reducir la probabilidad de enfren- } \\
\text { tar perturbaciones repentinas, resistir la propagación de las perturbaciones manteniendo el control sobre } \\
\text { las estructuras y funciones, y recuperarse y responder mediante planes reactivos inmediatos y efectivos } \\
\text { para trascender la perturbación y restaurar la cadena de suministro a un estado robusto de operaciones } \\
\text { (Pires Ribeiro y Barbosa-Povoa 2018) }\end{array}$ \\
\hline
\end{tabular}

\section{Metodología}

La revisión de la literatura es parte de la estructura básica de una investigación, en este sentido el objetivo de este estudio es analizar sistemáticamente las revisiones realizadas sobre la cadena suministro teniendo en cuenta la relación con el enfoque aplicado, identificando sus elementos clave y los tipos de métodos estudiados hasta la fecha. En este apartado se da respuesta a la PI2.

Para el cumplimiento de este objetivo se utiliza la metodología planteada por Seuring y Müller (2008) y Seuring y Gold (2012) en la que se establecen cuatro pasos para realizar un revisión sistemática: 1) recolección del material, 2) análisis descriptivo, 3) selección de categorías y 4) evaluación del material.

\subsection{Recolección}

El alcance del estudio empieza con la selección de las bases de datos, siendo SCOPUS y Web Of Science las seleccionadas. Los criterios de búsqueda utilizados se muestran en la Tabla 2. Estos criterios de búsqueda fueron adaptados a cada base de datos específica para garantizar la solidez de la búsqueda. 
Tabla 2 Criterios de búsqueda en las bases de datos

\begin{tabular}{ll}
\hline Identificación & Descripción \\
\hline Bases de datos & SCOPUS, Web Of Science \\
\hline Lenguaje & inglés \\
\hline Áreas Científicas & todas \\
\hline Revistas & todas \\
\hline Tipos de artículos & revisión de la literatura \\
\hline Campo de búsqueda & título, resumen y palabras clave \\
\hline & \\
Fecha de búsqueda & febrero 2019 \\
\hline & 2009-2019 \\
\hline
\end{tabular}

Con el fin de garantizar la selección adecuada de artículos, la búsqueda se limita a revistas en inglés, excluyendo las revisiones de conferencias, libros etc. Además, la búsqueda se acota aplicando las palabras claves basadas en las preguntas de investigación (ver figura 1).

El periodo de búsqueda se ajusta a los últimos 10 años, pues el propósito de la presente investigación es conocer qué líneas futuras de investigación (para la próxima déca- da) plantean los autores. Por otro lado, varios de los autores estudiados (Brandenburg et al. 2014; Malviya y Kant 2015; Barbosa-Póvoa, da Silva, y Carvalho 2018; Ho et al. 2015) analizan revisiones de la literatura, además de trabajos primarios. En concordancia a los investigadores mencionados, Brandenburg et al. (2014) analizan revisiones desde 1999 al 2012, Ho et al. (2015) desde 2006 al 2012, Malviya y Kant (2015) desde 2007 al 2013, Barbosa-Póvoa et al. (2018) desde el 2007 al 2016.

Figura 1 Criterios de búsqueda y selección de artículos

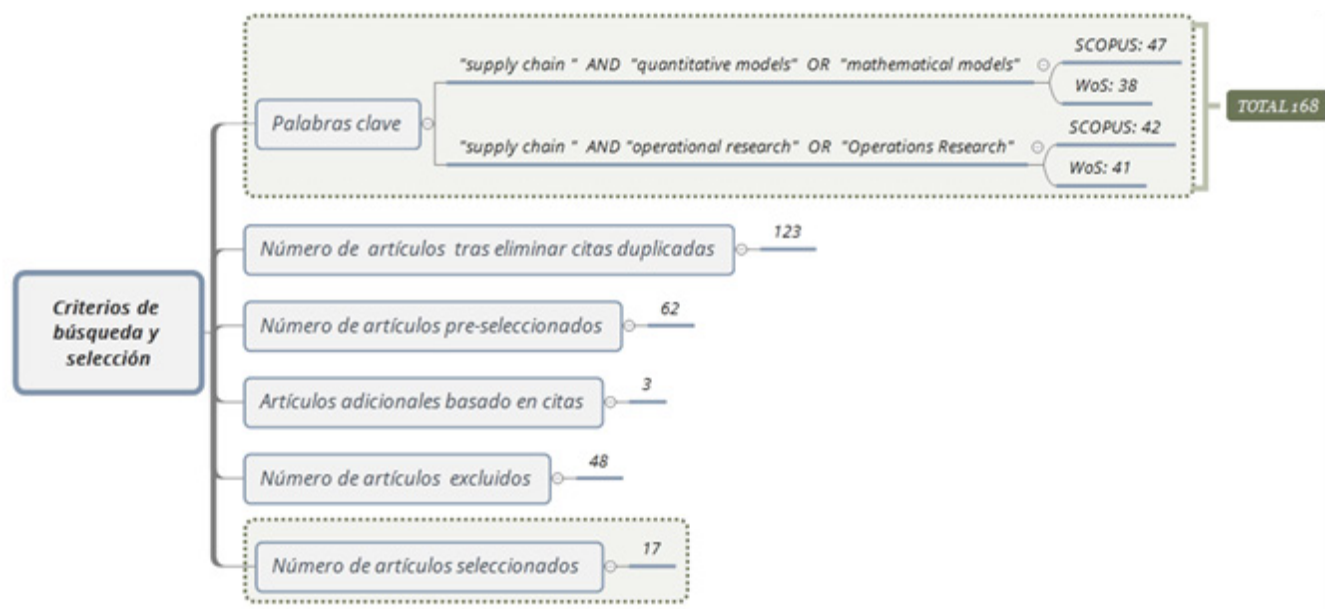

La Figura 1 resume el proceso de selección de las revisiones de la literatura consideradas en este estudio: de la primera búsqueda en las bases de datos seleccionadas se obtuvieron un total de 168 referencias, después eliminando duplicados quedaron 123 artículos. La lectura del resumen (abstract) de los 123 artículos permitió evaluar si los artículos se ajustan a nuestras preguntas de investigación. Aplicando este criterio el número de artículos se redujo a 62, los cuales fueron considerados para su posterior revisión. El texto completo de estos 62 artículos fue revisado con profundidad analizando su respuesta a las siguientes preguntas: ¿el estudio presenta modelos o métodos de resolución de problemas de optimización en la cadena de suministro?; ¿el estudio describe una relación con planes de aprovisio- namiento, producción y distribución?; ¿el estudio describe una perspectiva y un sector de aplicación?; ¿el estudio propone líneas de investigación futuras? Después de evaluar estas investigaciones se identificaron 3 artículos que fueron citados, pero no fueron capturados por nuestros criterios de búsqueda, estos 3 artículos no estaban agrupados como revisiones de literatura, sino que estaban agrupados como artículos. Después de leer estos artículos identificamos su relevancia y evaluamos si se ajustan a nuestras preguntas de investigación. La recolección arrojo 65 artículos los cuales fueron analizados en profundidad y posteriormente fueron excluidos 48 artículos porque no se ajustaban adecuadamente a nuestras preguntas de investigación, finalmente seleccionamos 17 para ser estudiados (véase Tabla 3). 
Tabla 3 Artículos de revisión seleccionados.

\begin{tabular}{|c|c|c|}
\hline Año & Autores & Título \\
\hline 2009 & $\begin{array}{l}\text { David Peidro, Josefa Mula, Raúl Poler, Francisco-Cruz } \\
\text { Lario }\end{array}$ & $\begin{array}{l}\text { Quantitative models for supply chain planning under un- } \\
\text { certainty: a review. }\end{array}$ \\
\hline 2010 & $\begin{array}{l}\text { Josefa Mula, David Peidro, Manuel Díaz-Madroñero, } \\
\text { Eduardo Vicens }\end{array}$ & $\begin{array}{l}\text { Mathematical programming models for supply chain pro- } \\
\text { duction and transport planning. }\end{array}$ \\
\hline 2014 & Dennis Stindt, Ramin Sahamie & $\begin{array}{l}\text { Review of research on closed loop supply chain manage- } \\
\text { ment in the process industry. }\end{array}$ \\
\hline 2014 & $\begin{array}{l}\text { Marcus Brandenburg, Kannan Govindan, Joseph Sark- } \\
\text { is, Stefan Seuring }\end{array}$ & $\begin{array}{l}\text { Quantitative models for sustainable supply chain man- } \\
\text { agement: Developments and directions. }\end{array}$ \\
\hline 2015 & Rakesh Kumar Malviya, Ravi Kant & $\begin{array}{l}\text { Green supply chain management (GSCM): a structured } \\
\text { literature review and research implications. }\end{array}$ \\
\hline 2015 & Çağrı Sel, Bilge Bilgen & $\begin{array}{l}\text { Quantitative models for supply chain management within } \\
\text { dairy industry: a review and discusion. }\end{array}$ \\
\hline 2015 & Kannan Govindan, Hamed Soleimani, Devika Kannan & $\begin{array}{l}\text { Reverse logistics and closed-loop supply chain: A com- } \\
\text { prehensive review to explore the future. }\end{array}$ \\
\hline 2015 & $\begin{array}{l}\text { William Ho, Tian Zheng, Hakan Yildiz, Srinivas Tal- } \\
\text { luric }\end{array}$ & Supply chain risk management: a literature review. \\
\hline 2016 & $\begin{array}{l}\text { Muhammad Salman Habib, Young Hae Lee, Muham- } \\
\text { mad Saad Memon }\end{array}$ & $\begin{array}{l}\text { Mathematical Models in Humanitarian Supply Chain } \\
\text { Management: A Systematic Literature Review. }\end{array}$ \\
\hline 2016 & $\begin{array}{l}\text { Wladimir E. Soto-Silva, Esteve Nadal-Roig, Marcela } \\
\text { C. González, Lluis M. Pla-Aragones }\end{array}$ & $\begin{array}{l}\text { Operational research models applied to the fresh fruit } \\
\text { supply chain. }\end{array}$ \\
\hline 2017 & Carlos Franco, Edgar Alfonso-Lizarazo & $\begin{array}{l}\text { A Structured Review of Quantitative Models of the Phar- } \\
\text { maceutical Supply Chain. }\end{array}$ \\
\hline 2018 & $\begin{array}{l}\text { Nasim Zandi Atashbar, Nacima Labadie, Christian } \\
\text { Prins }\end{array}$ & $\begin{array}{l}\text { Modelling and optimisation of biomass supply chains: a } \\
\text { review. }\end{array}$ \\
\hline 2018 & $\begin{array}{l}\text { Faiza Hamdi, Ahmed Ghorbel, Faouzi Masmoudi, Li- } \\
\text { onel Dupont }\end{array}$ & $\begin{array}{l}\text { Optimization of a supply portfolio in the context of sup- } \\
\text { ply chain risk management: literature review. }\end{array}$ \\
\hline 2018 & Cigdem Gonul Kochan, David R. Nowicki & $\begin{array}{l}\text { Supply chain resilience: a systematic literature review } \\
\text { and typological framework. }\end{array}$ \\
\hline 2018 & $\begin{array}{l}\text { Ana Paula Barbosa-Póvoa, Cátia da Silva, Ana Carval- } \\
\text { ho }\end{array}$ & $\begin{array}{l}\text { Opportunities and challenges in sustainable supply chain: } \\
\text { An operations research perspective. }\end{array}$ \\
\hline 2019 & $\begin{array}{l}\text { Isabel Mundi, M. M. E. Alemany, Raúl Poler \& Vicente } \\
\text { S. Fuertes-Miquel }\end{array}$ & $\begin{array}{l}\text { Review of mathematical models for production planning } \\
\text { under uncertainty due to lack of homogeneity: proposal } \\
\text { of a conceptual model. }\end{array}$ \\
\hline 2019 & $\begin{array}{l}\text { J.B. Oliveiraa, M. Jin, R.S. Lima, J.E. Kobza, J.A.B. } \\
\text { Montevechia }\end{array}$ & $\begin{array}{l}\text { The role of simulation and optimization methods in sup- } \\
\text { ply chain risk management: Performance and review } \\
\text { standpoints. }\end{array}$ \\
\hline
\end{tabular}

\subsection{Análisis descriptivo}

En esta fase se analizan los 17 artículos seleccionados con el fin de responder a la PI3. Las características que se evaluaron son: fecha de la publicación, tipo de publicación y la revista. Entre las revistas a las que pertenecen las publicaciones, destacan European Journal of Operational Research e International Journal of Production Research (Tabla 4). 
Tabla 4 Distribución de artículos en base a las revistas

\begin{tabular}{ll}
\hline Revista & Artículos n=17 \\
\hline European Journal of Operational Research & 5 \\
\hline International Journal of Production Research & 3 \\
\hline Mathematical Problems in Engineering & 1 \\
\hline Benchmarking: An International Journal & 1 \\
\hline Complexity & 1 \\
\hline European J. Industrial Engineering, & 1 \\
\hline Flexible Services and Manufacturing Journal & 1 \\
\hline $\begin{array}{l}\text { The International Journal of Advanced Manufacturing Technol- } \\
\text { ogy }\end{array}$ & 1 \\
\hline $\begin{array}{l}\text { International Journal of Physical Distribution \& Logistics Man- } \\
\text { agement }\end{array}$ & 1 \\
\hline Journal of Intelligent Manufacturing & 1 \\
\hline Simulation Modelling Practice and Theory & 1 \\
\hline
\end{tabular}

años y es destacable el hecho de que el $27,8 \%$ se publicaron en 2018. El año 2015 es de igual forma significativo con un $22,2 \%$, mientras que el resto fechas representa menos del $11,1 \%$ como lo muestra la Figura 2.
Analizando las fechas de publicación se obtiene información sobre la evolución de las investigaciones que se han realizado en las diferentes perspectivas de la cadena de suministro. Los artículos seleccionados cubren un periodo de 10
Figura 1 Clasificación de artículos

según el año de publicación.

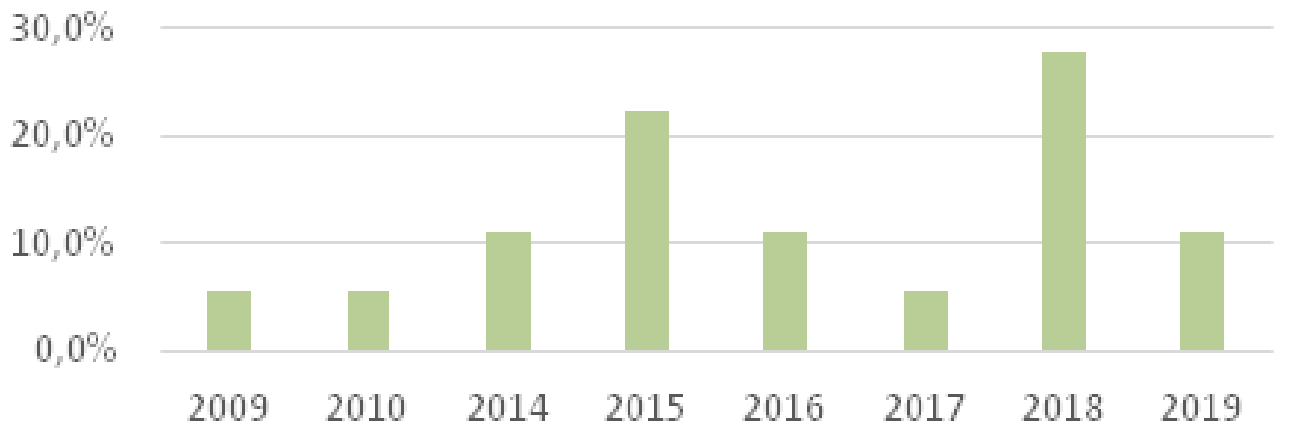

\subsection{Selección de categorías}

Los principales temas de análisis y las dimensiones estructurales que se incluyen en esta selección están descritas en las Tablas 5 y 6 . Los procesos que se realizan en la cadena de suministro pueden ser clasificados a través del modelo SCOR (Supply Chain Operations Reference), enfoque que describe los procesos de los negocios clasificándolos en 3 categorías: Aprovisionamiento (S, Source), Producción/Manufactura/ Fabricación (M, Make) y Distribución (D, Deliver), a los que se añaden subtipos y esquemas (Andres et al., 2017b). Con esta categorización se resuelve la PI4 (Tabla 5). 
Tabla 5 Tipos y subtipos de planes

según el modelo SCOR

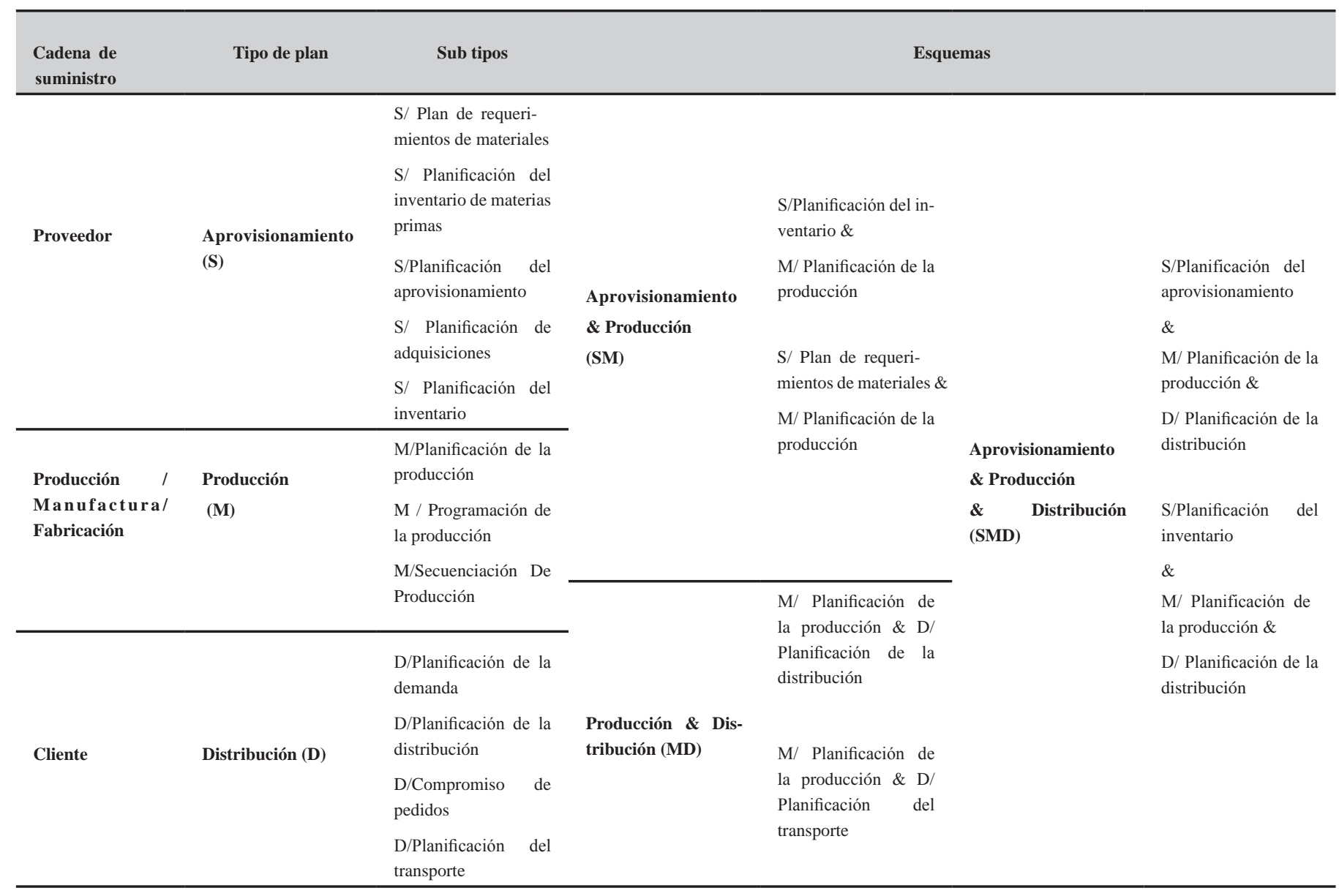

(Andres et al., 2017a) proponen una clasi icación de modelos y algoritmos utilizados para resolver planes de aprovisionamiento, producción y distribución en el marco del proyecto Europeo "Redes Colaborativas de Fabricación en Nube” (Cloud Collaborative Manufacturing Networks
- C2NET), presentada en el entregable "Taxonomy of optimisation and simulation solutions for Manufacturing and Logistics Processes” (C2NET 2016). La Tabla 6 responde a la PI5 y muestra esta clasificación y otros parámetros considerados en la evaluación de las revisiones. 
Tabla 6 Clasificación de categorías de acuerdo con las taxonomías propuestas por (Brandenburg et al. 2014; Kochan y Nowicki 2018; C2NET 2016)

\begin{tabular}{ll}
\hline Tipos de modelos & Analíticos, Simulación, Matemáticos. \\
\hline Analíticos & Toma de decisiones multi-criterio (multi-criteria decision making - MCDM); Proceso de jerarquía Analíti- \\
& ca (Analytic Hierarchical Process - AHP); Proceso Analítico en Red (Analytic Network Process - ANP); \\
& Técnica para el Orden de Preferencia por Similitud con la Solución Ideal (Technique for Order of Prefer- \\
& ence by Similarity to Ideal Solution - TOPSIS); Eliminación y elección expresando la realidad (Elimina- \\
& tion and Choice Expressing Reality - ELECTRE); Optimización multicriterio y solución de compromiso \\
& (Multicriteria Optimization and Compromise Solution - VIKOR)
\end{tabular}

\begin{tabular}{|c|c|c|}
\hline Simulación & \multicolumn{2}{|c|}{$\begin{array}{l}\text { Simulación basada en agentes (Agent-Based Simulation - ABS); Simulación de eventos discretos (Discrete } \\
\text { Event Simulation - DES); Simulación de dinámica de Sistemas (System Dynamics Simulation - SDS); } \\
\text { Simulación Monte Carlo (Monte Carlo Simulation); Otros métodos de simulación }\end{array}$} \\
\hline Matemáticos & \multicolumn{2}{|c|}{$\begin{array}{l}\text { Programación Lineal (Linear Programming - LP); Programación Lineal Entera (Integer Linear Program- } \\
\text { ming - ILP); Programación Lineal Entera Mixta (Mixed Integer Linear Programming - MILP); Progra- } \\
\text { mación no lineal /Entera Mixta / no lineal entera (Non-Linear Programming - NLP) /Mixed integer/In- } \\
\text { teger non linear programming); Programación cuadrática (Quadratic Programming - QP); Programación } \\
\text { dinámica (Dynamic Programming - DP); Programación estocástica (Stochastic Programming - SP); Pro- } \\
\text { gramación robusta (Robust Programming - RP); Programación por restricciones (Constraint Programming } \\
\text { - CP); Programación lineal multi-objetivo / Programación no lineal multi-objetivo/programación no lineal } \\
\text { entera mixta multi-objetivo (Multi-Objective Linear Programming - MOLP) / Multi-Objective Non-Linear } \\
\text { Problem - MONLP) /Multi-Objective Mixed-Integer Non-Linear Programming - MOMINLP); } \\
\text { Enfoques híbridos (Hybrid Approach - HA); Programación Difusa (Fuzzy Programming - FP); Progra- } \\
\text { mación por Objetivos (Goal Programming - GP) }\end{array}$} \\
\hline Métodos de resolución & \multicolumn{2}{|c|}{ Algoritmos Optimizadores (AO), Heurísticos (AH), Metaheurísticos (AM) } \\
\hline Optimizadores (AO) & Heurísticos (AH) & Metaheurísticos (AM) \\
\hline AO/ Descomposición & AH/ Planificación Maestra Multi-Objetivo & AM/Optimización de Colonias \\
\hline AO/ Lomnicki & AH/ Campbell-Dudeck & AM/ Computación evolutiva \\
\hline \multirow{2}{*}{$\begin{array}{l}\text { AO/ Optimización es- } \\
\text { tratégica-operativa }\end{array}$} & AH/ Mejora Local & AM/ Genéticos \\
\hline & AH/ Heurística Primal-Dual & AM/GRASP \\
\hline AO/Branch and Bound & AH/ Descomposición y Agregados & AM/Búsqueda local iterativa \\
\hline $\mathrm{AO} /$ Criss-cross & AH/Greedy & AM/Redes Neuronales \\
\hline AO/ Lompen & AH/Lagrangiana & AM/Búsqueda dispersa \\
\hline \multirow[t]{4}{*}{ AO/Simplex } & AH/ Árbol recubridor mínimo & AM/ Recocido Simulado \\
\hline & AH/ Vecino más cercano & AM/ Búsqueda Tabú \\
\hline & & AM/TSGW \\
\hline & & AM/ Búsqueda de vecindad variable \\
\hline
\end{tabular}

\subsection{Validación}

En esta fase se realizó la validación de los artículos analizados de acuerdo con las características, enfoques y dimensiones seleccionadas utilizando la técnica de agrupamiento jerárquico aglomerativo a través de un proceso deductivo e inductivo.

La evaluación se ocupó de que las revisiones fueran apropiadas y que contaran con información suficiente, condicionando de esta forma, que los artículos se sujeten a los parámetros considerados para la clasificación, siendo esta una de las razones por las que varias publicaciones fueron descartadas. El análisis ayudó a que la organización, categorización, estructura, y los principales hallazgos de la revisión sistemática se sometieran a un examen, motivando con esto a encontrar futuras líneas de investigación.

\section{Resultados}

En esta sección se describen los resultados obtenidos de la categorización y evaluación de los artículos de revisión seleccionados, analizando elementos como los tipos de planes utilizados, los modelos y los métodos de resolución.

\subsection{Perspectivas de análisis}

Los artículos de revisión seleccionados presentan diferentes perspectivas de análisis: gestión de la cadena de suministro, cadena de suministro bajo incertidumbre, gestión cadena de suministro de ciclo cerrado, gestión sostenible de la cadena de suministro, gestión de riesgos de la cadena de suministro, gestión de la cadena de suministro verde, resiliencia de la cadena de suministro. Estas investigaciones 
Estas investigaciones analizaron un total de 2309 artículos, teniendo un periodo aproximado de búsqueda que va desde 1983 hasta el 2019. La Tabla 7 muestra cuales son los sectores en los que estos estudios se centraron, así, varias investigaciones se centraron en un sector específico estudiando todas aquellas publicaciones que se refieran al sector en relación con la perspectiva analizada, otras revisiones analizaron todos los sectores bajo la perspectiva de análisis. Es importante destacar que la mayor cantidad de artículos analizados por los estudios de revisión se concentran entre el año 2007 y el 2013, representado este periodo un 60,64\% los artículos (Figura 3), siendo el año 2012 el que aglomera mayor cantidad de artículos con 241 (10,75\%).

Otro aspecto analizado por las revisiones son niveles de decisión en el proceso de la planificación, las investigaciones analizan los niveles: estratégico, táctico y operativo. La Tabla 7 indica qué estudios consideraron este aspecto.

Figura 3 Número de artículos publicados por año citados en los artículos de revisión analizados.

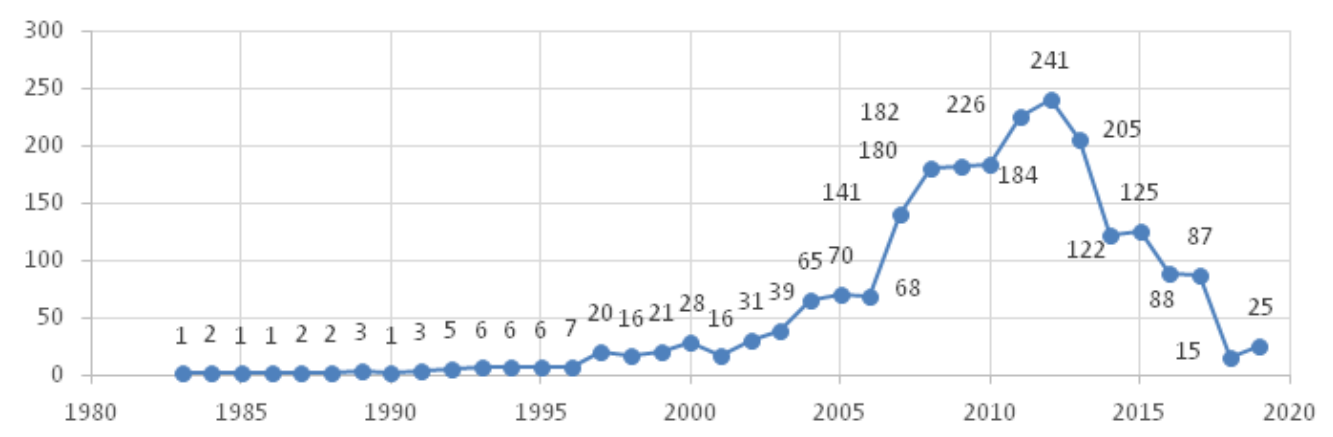

Tabla 7 Análisis del enfoque de las investigaciones realizadas

\begin{tabular}{|c|c|c|c|c|c|c|c|}
\hline Autor & Revista & $\begin{array}{l}\text { Artículos } \\
\text { revisados }\end{array}$ & $\begin{array}{l}\text { Periodo de } \\
\text { análisis }\end{array}$ & $\begin{array}{l}\text { Pers pectiva } \\
\text { analizada }\end{array}$ & $\begin{array}{l}\text { Estudio de nive- } \\
\text { les de decisión }\end{array}$ & Sector analizado & Enfoque del estudio \\
\hline $\begin{array}{l}\text { (Peidro et } \\
\text { al. 2009) }\end{array}$ & $\begin{array}{l}\text { The International } \\
\text { Journal of Ad- } \\
\text { vanced Manufac- } \\
\text { turing Technology }\end{array}$ & 103 & $1988-2007$ & $\begin{array}{l}\text { Cadena de sumi- } \\
\text { nistro bajo incer- } \\
\text { tidumbre }\end{array}$ & $\mathrm{x}$ & Multisectorial & $\begin{array}{l}\text { Se centra en la planificación } \\
\text { de la cadena de suministro en } \\
\text { condiciones de incertidumbre } \\
\text { mediante la adopción de enfo- } \\
\text { ques cuantitativos. }\end{array}$ \\
\hline $\begin{array}{l}\text { (Mula et } \\
\text { al. 2010) }\end{array}$ & $\begin{array}{l}\text { European Journal } \\
\text { of Operational } \\
\text { Research }\end{array}$ & 44 & 1989-2009 & $\begin{array}{l}\text { Cadena de sumi- } \\
\text { nistro }\end{array}$ & $\mathrm{x}$ & Multisectorial & $\begin{array}{l}\text { Estudia los modelos de pro- } \\
\text { gramación matemática para } \\
\text { la producción de la cadena de } \\
\text { suministro y la planificación } \\
\text { del transporte, basados en el } \\
\text { análisis de ocho aspectos: es- } \\
\text { tructura, nivel de decisión, en- } \\
\text { foque de modelado, propósito, } \\
\text { información compartida, lim- } \\
\text { itaciones del modelo, la nove- } \\
\text { dad aportada y la aplicación } \\
\text { práctica. }\end{array}$ \\
\hline $\begin{array}{l}\text { (Stindt y } \\
\text { Sahamie } \\
\text { 2014) }\end{array}$ & $\begin{array}{l}\text { Flexible Services } \\
\text { and Manufactur- } \\
\text { ing Journal }\end{array}$ & 167 & $1994-2012$ & $\begin{array}{l}\text { Gestión de cadena } \\
\text { de suministro de } \\
\text { ciclo cerrado }\end{array}$ & - & $\begin{array}{l}\text { Química, Indus- } \\
\text { tria del metal / } \\
\text { acero, Industria } \\
\text { de la construc- } \\
\text { ción, Industria } \\
\text { del papel /pulpa, } \\
\text { Industria far- } \\
\text { macéutica, Indus- } \\
\text { tria de plásticos / } \\
\text { polímeros, Indus- } \\
\text { tria textil }\end{array}$ & $\begin{array}{l}\text { Describe enfoques cuantita- } \\
\text { tivos en la planificación de la } \\
\text { cadena de suministro de ciclo } \\
\text { cerrado en la industria de pro- } \\
\text { cesos }\end{array}$ \\
\hline
\end{tabular}




\begin{tabular}{|c|c|c|c|c|c|c|c|}
\hline Autor & Revista & $\begin{array}{l}\text { Artículos } \\
\text { revisados }\end{array}$ & $\begin{array}{l}\text { Periodo de } \\
\text { análisis }\end{array}$ & $\begin{array}{l}\text { P e r s p e c t iv a } \\
\text { analizada }\end{array}$ & $\begin{array}{l}\text { Estudio de nive- } \\
\text { les de decisión }\end{array}$ & Sector & Enfoque del estudio \\
\hline $\begin{array}{l}\text { (Branden- } \\
\text { burg et al. } \\
\text { 2014) }\end{array}$ & $\begin{array}{l}\text { European Journal } \\
\text { of Operational } \\
\text { Research }\end{array}$ & 134 & 1994-2012 & $\begin{array}{l}\text { Gestión sosteni- } \\
\text { ble de su cadena } \\
\text { de suministro }\end{array}$ & - & Multisectorial & $\begin{array}{l}\text { Estudia modelos cuantitativos } \\
\text { para la gestión sostenible de la } \\
\text { cadena de suministro. }\end{array}$ \\
\hline $\begin{array}{l}\text { (Malviya } \\
\text { y Kant } \\
2015)\end{array}$ & $\begin{array}{l}\text { Benchmarking: } \\
\text { An International } \\
\text { Journal }\end{array}$ & 177 & 1998-2013 & $\begin{array}{l}\text { Gestión de la ca- } \\
\text { dena de suminis- } \\
\text { tro verde }\end{array}$ & - & Multisectorial & $\begin{array}{l}\text { Identificó y analizó los métodos } \\
\text { de investigación, las técnicas de } \\
\text { análisis de datos, los métodos } \\
\text { de toma de decisiones multicri- } \\
\text { terio y las principales industrias } \\
\text { que participan activamente en } \\
\text { la gestión de la cadena de sum- } \\
\text { inistro verde. }\end{array}$ \\
\hline $\begin{array}{l}\text { (Sel y Bil- } \\
\text { gen 2015) }\end{array}$ & $\begin{array}{l}\text { European J. In- } \\
\text { dustrial Engineer- } \\
\text { ing, }\end{array}$ & 78 & $1983-2013$ & $\begin{array}{l}\text { Gestión de la } \\
\text { cadena de sumi- } \\
\text { nistro }\end{array}$ & $\mathrm{x}$ & Lácteo & $\begin{array}{l}\text { Explora los modelos cuanti- } \\
\text { tativos para la planificación y } \\
\text { programación de la producción, } \\
\text { planificación de la distribución } \\
\text { y problemas de enrutamiento } \\
\text { de vehículos (VRP) dentro de } \\
\text { la gestión de la cadena de sum- } \\
\text { inistro de los productos lácteos. }\end{array}$ \\
\hline $\begin{array}{l}\text { (Govin- } \\
\text { dan et al., } \\
\text { 2015) }\end{array}$ & $\begin{array}{l}\text { European Journal } \\
\text { of Operational } \\
\text { Research }\end{array}$ & 382 & $2007-2013$ & $\begin{array}{l}\text { Cadena de sum- } \\
\text { inistro de ciclo } \\
\text { cerrado }\end{array}$ & $\mathrm{x}$ & Multisectorial & $\begin{array}{l}\text { Plasma una revisión de la } \\
\text { literatura de métodos de op- } \\
\text { timización utilizados en la } \\
\text { logística inversa y en la cadena } \\
\text { de suministro de ciclo cerrado }\end{array}$ \\
\hline $\begin{array}{l}\text { (Ho et al. } \\
\text { 2015) }\end{array}$ & $\begin{array}{l}\text { International } \\
\text { Journal of Pro- } \\
\text { duction Research }\end{array}$ & 159 & $2003-2013$ & $\begin{array}{l}\text { Gestión del riesgo } \\
\text { de la cadena de } \\
\text { suministro }\end{array}$ & - & Multisectorial & $\begin{array}{l}\text { Examina los métodos cuantita- } \\
\text { tivos y cualitativos de gestión } \\
\text { de riesgos de la cadena de } \\
\text { suministro de acuerdo con } \\
\text { cuatro procesos principales: } \\
\text { identificación, la evaluación, } \\
\text { la mitigación y el monitoreo de } \\
\text { riesgos }\end{array}$ \\
\hline $\begin{array}{l}\text { (Habib et } \\
\text { al., 2016) }\end{array}$ & $\begin{array}{l}\text { Math e m a tic a l } \\
\text { Problems in Engi- } \\
\text { neering }\end{array}$ & 140 & 2005-2015 & $\begin{array}{l}\text { Gestión de la } \\
\text { cadena de sumi- } \\
\text { nistro }\end{array}$ & - & Humanitaria & $\begin{array}{l}\text { Indaga sobre las técnicas de } \\
\text { optimización matemática y los } \\
\text { algoritmos desarrollados en las } \\
\text { operaciones humanitarias de la } \\
\text { cadena de suministro. }\end{array}$ \\
\hline $\begin{array}{l}\text { (Soto-Sil- } \\
\text { va et al. } \\
2016)\end{array}$ & $\begin{array}{l}\text { European Journal } \\
\text { of Operational } \\
\text { Research }\end{array}$ & 28 & $1976-2015$ & $\begin{array}{l}\text { Cadena de sumi- } \\
\text { nistro }\end{array}$ & $\mathrm{x}$ & Frutas frescas & $\begin{array}{l}\text { Se centra en modelos de inves- } \\
\text { tigación operativa para resolver } \\
\text { problemas de decisión relacio- } \\
\text { nados con la cadena de sumi- } \\
\text { nistro de fruta fresca. }\end{array}$ \\
\hline $\begin{array}{l}\text { (Franco } \\
\text { y Alfon- } \\
\text { so-Lizara- } \\
\text { zo 2017) }\end{array}$ & Complexity & 46 & 1984-2016 & $\begin{array}{l}\text { Cadena de sumi- } \\
\text { nistro }\end{array}$ & $\mathrm{x}$ & Farmacéutico & $\begin{array}{l}\text { Analiza el diseño de redes, los } \\
\text { modelos de inventario y la op- } \\
\text { timización de una cadena de } \\
\text { suministro farmacéutica. }\end{array}$ \\
\hline $\begin{array}{l}\text { ( Z a n d i } \\
\text { A t a s h- } \\
\text { bar et al. } \\
\text { 2018) }\end{array}$ & $\begin{array}{l}\text { International } \\
\text { Journal of Pro- } \\
\text { duction Research }\end{array}$ & 110 & 1996-2017 & $\begin{array}{l}\text { Cadena de sumi- } \\
\text { nistro }\end{array}$ & $\mathrm{x}$ & Biomasa & $\begin{array}{l}\text { Describe modelos, métodos y } \\
\text { enfoques de solución para opti- } \\
\text { mizar las cadenas de suministro } \\
\text { de biomasa. }\end{array}$ \\
\hline $\begin{array}{l}\text { (Hamdi et } \\
\text { al. 2018) }\end{array}$ & $\begin{array}{l}\text { Journal of Intel- } \\
\text { ligent Manufac- } \\
\text { turing }\end{array}$ & 124 & $2003-2014$ & $\begin{array}{l}\text { Gestión del riesgo } \\
\text { de la cadena de } \\
\text { suministro }\end{array}$ & - & Multisectorial & $\begin{array}{l}\text { Describe las técnicas de opti- } \\
\text { mización y el enfoque cuantita- } \\
\text { tivo, cualitativo e híbrido en la } \\
\text { gestión de riesgos de la cadena } \\
\text { de suministro. }\end{array}$ \\
\hline
\end{tabular}




\begin{tabular}{|c|c|c|c|c|c|c|c|}
\hline Autor & Revista & $\begin{array}{l}\text { Artículos } \\
\text { revisados }\end{array}$ & $\begin{array}{l}\text { Periodo de } \\
\text { análisis }\end{array}$ & $\begin{array}{l}\text { P e r s p e c t i v a } \\
\text { analizada }\end{array}$ & $\begin{array}{l}\text { Estudio de nive- } \\
\text { les de decisión }\end{array}$ & Sector & Enfoque del estudio \\
\hline $\begin{array}{l}\text { (Kochan y } \\
\text { Nowicki } \\
\text { 2018) }\end{array}$ & $\begin{array}{l}\text { International } \\
\text { Journal of Phys- } \\
\text { ical Distribution } \\
\text { \& Logistics Man- } \\
\text { agement }\end{array}$ & 228 & $2003-2017$ & $\begin{array}{l}\text { Resiliencia de la } \\
\text { cadena de sumi- } \\
\text { nistro }\end{array}$ & - & Multisectorial & $\begin{array}{l}\text { Analizó métodos cuantitativos } \\
\text { y de simulación y reviso las } \\
\text { definiciones relacionadas con } \\
\text { la resiliencia de la cadena de } \\
\text { suministro e identifica medidas } \\
\text { y técnicas de evaluación. }\end{array}$ \\
\hline $\begin{array}{l}\text { (B a rbo- } \\
\text { sa-Póvoa } \\
\text { et al. } \\
\text { 2018) }\end{array}$ & $\begin{array}{l}\text { European Journal } \\
\text { of Operational } \\
\text { Research }\end{array}$ & 220 & $2007-2016$ & $\begin{array}{l}\text { Cadena de sumi- } \\
\text { nistro sostenible }\end{array}$ & $\mathrm{x}$ & Multisectorial & $\begin{array}{l}\text { Refiere los métodos de inves- } \\
\text { tigación operativa para apoyar } \\
\text { actividades de cadena de sum- } \\
\text { inistro sostenibles. }\end{array}$ \\
\hline $\begin{array}{l}\text { (Mundi et } \\
\text { al. 2019) }\end{array}$ & $\begin{array}{l}\text { International } \\
\text { Journal of Pro- } \\
\text { duction Research }\end{array}$ & 87 & $1995-2018$ & $\begin{array}{l}\text { Cadena de sumi- } \\
\text { nistro bajo incer- } \\
\text { tidumbre }\end{array}$ & $\mathrm{x}$ & $\begin{array}{l}\text { Petróleo / Agroal- } \\
\text { imentario / Re- } \\
\text { manufactura / } \\
\text { Sector madera / } \\
\text { Sector minero / } \\
\text { Cerámico }\end{array}$ & $\begin{array}{l}\text { Analiza modelos matemáti- } \\
\text { cos para la planificación de la } \\
\text { producción bajo incertidumbre } \\
\text { dada la falta de homogeneidad. }\end{array}$ \\
\hline $\begin{array}{l}\text { (Olivei- } \\
\text { ra et al. } \\
2019)\end{array}$ & $\begin{array}{l}\text { Simulation Mod- } \\
\text { elling Practice } \\
\text { and Theory }\end{array}$ & 52 & $2000-2017$ & $\begin{array}{l}\text { Gestión del riesgo } \\
\text { de la cadena de } \\
\text { suministro }\end{array}$ & - & Multisectorial & $\begin{array}{l}\text { Investiga los métodos de sim- } \\
\text { ulación y optimización para el } \\
\text { enfoque de gestión de riesgos } \\
\text { de la cadena de suministro }\end{array}$ \\
\hline
\end{tabular}

\subsection{Procesos de la cadena de suministro}

Los trabajos seleccionados enfocaron su análisis en tratar temas relacionados con el aprovisionamiento, producción y distribución. Muchos de estos artículos han conceptualizado, categorizado y descrito los planes y los modelos planteados en los procesos de planificación de la cadena de suministro.
No obstante, cada publicación presenta su propia metodología y criterio de clasificación. Con el objeto de presentar un cuadro comparativo, se clasifican los procesos de planificación analizados a las categorías del modelo SCOR, como muestra la Tabla 8.
Tabla 8 Clasificiacion según modelos SCOR

\begin{tabular}{|c|c|c|c|}
\hline & & Proceso de & la de suministro \\
\hline Autor & $\begin{array}{c}\text { Aprovisionamiento } \\
\text { (Source) }\end{array}$ & $\begin{array}{c}\text { Producción } \\
\text { (Make) }\end{array}$ & $\begin{array}{c}\text { Transporte } \\
\text { (Deliver) }\end{array}$ \\
\hline (Peidro et al. 2009) & $\mathrm{x}$ & $\mathrm{x}$ & $\mathrm{x}$ \\
\hline (Mula et al. 2010) & $\mathrm{x}$ & $\mathrm{x}$ & $\mathrm{x}$ \\
\hline (Stindt y Sahamie 2014) & - & $\mathrm{x}$ & - \\
\hline (Brandenburg et al. 2014) & $\mathrm{x}$ & $\mathrm{x}$ & $\mathrm{x}$ \\
\hline (Malviya y Kant 2015) & $\mathrm{x}$ & $\mathrm{x}$ & $\mathrm{x}$ \\
\hline (Sel y Bilgen 2015) & $\mathrm{x}$ & $\mathrm{x}$ & $\mathrm{x}$ \\
\hline (Govindan, Soleimani, y Kannan 2015) & $\mathrm{x}$ & $\mathrm{x}$ & $\mathrm{x}$ \\
\hline (Ho et al. 2015) & $\mathrm{x}$ & $\mathrm{x}$ & $\mathrm{x}$ \\
\hline (Habib, Lee, y Memon 2016) & $\mathrm{x}$ & - & $\mathrm{x}$ \\
\hline (Soto-Silva et al. 2016) & $\mathrm{x}$ & $\mathrm{x}$ & $\mathrm{x}$ \\
\hline (Franco y Alfonso-Lizarazo 2017) & $\mathrm{x}$ & $\mathrm{x}$ & $\mathrm{x}$ \\
\hline
\end{tabular}




\begin{tabular}{|c|c|c|c|}
\hline (Zandi Atashbar, Labadie, y Prins 2018) & $\mathrm{x}$ & $\mathrm{x}$ & $\mathrm{x}$ \\
\hline (Hamdi et al. 2018) & $\mathrm{x}$ & $\mathrm{x}$ & $\mathrm{x}$ \\
\hline (Kochan y Nowicki 2018) & - & - & - \\
\hline (Barbosa-Póvoa, da Silva, y Carvalho 2018) & $\mathrm{x}$ & $\mathrm{x}$ & $\mathrm{x}$ \\
\hline (Mundi et al. 2019) & $\mathrm{x}$ & $\mathrm{x}$ & $x$ \\
\hline (Oliveira et al. 2019) & $\mathrm{x}$ & $\mathrm{x}$ & $\mathrm{x}$ \\
\hline
\end{tabular}

\subsection{Enfoques de modelado}

De manera análoga, se clasificaron los modelos utilizados para resolver los problemas de optimización con el fin de responder a la PI6, obteniendo como resultado que los modelos analíticos (AHP, ANP, TOPSIS, ELECTRE, VIKOR) han sido analizados por la mayoría de los investigadores. Lo mismo ocurre con los métodos de simulación detallados en la Tabla 6. Mientras que los enfoques de modelado matemáticos más utilizados son los métodos de programación lineal, programación lineal entera mixta, programación no lineal, programación esto- cástica, programación multiobjetivo con sus variantes y la programación difusa. La Tabla 9 describe los tipos de modelos analizados en las revisiones.

En términos generales los investigadores utilizan diferentes solucionadores, para resolver los modelos, como CPLEX o Gurobi, no obstante pocos estudios consideraron este aspecto en sus revisiones (Stindt y Sahamie 2014; Habib, Lee, y Memon 2016; Zandi Atashbar, Labadie, y Prins 2018; Oliveira et al. 2019).

Tabla 9 Tipos de modelos encontrados

en las revisones.

\begin{tabular}{|c|c|c|c|c|c|c|c|c|c|c|c|c|c|c|c|}
\hline \multirow{2}{*}{ Autor } & \multicolumn{15}{|c|}{ Tipos de modelos } \\
\hline & A & $\mathrm{S}$ & 1 & 2 & 3 & 4 & 5 & 6 & 7 & 8 & 9 & 10 & 11 & 12 & 13 \\
\hline (Peidro et al. 2009) & $\mathrm{x}$ & $\mathrm{x}$ & $\mathrm{x}$ & $\mathrm{x}$ & $\mathrm{x}$ & $\mathrm{x}$ & & $\mathrm{x}$ & $\mathrm{x}$ & & & $\mathrm{x}$ & $\mathrm{x}$ & $\mathrm{x}$ & \\
\hline (Mula et al. 2010) & & $\mathrm{x}$ & $\mathrm{x}$ & $\mathrm{x}$ & $\mathrm{x}$ & $\mathrm{x}$ & & & $\mathrm{x}$ & & & $\mathrm{x}$ & $\mathrm{x}$ & $\mathrm{x}$ & \\
\hline (Stindt y Sahamie 2014) & $\mathrm{x}$ & & $\mathrm{x}$ & & $\mathrm{x}$ & & & & $\mathrm{x}$ & & & & $\mathrm{x}$ & & \\
\hline (Brandenburg et al. 2014) & $\mathrm{x}$ & $\mathrm{x}$ & $\mathrm{x}$ & & $\mathrm{x}$ & $\mathrm{x}$ & & $\mathrm{x}$ & $\mathrm{x}$ & & & & $\mathrm{x}$ & & $\mathrm{x}$ \\
\hline (Malviya y Kant 2015) & $\mathrm{x}$ & $\mathrm{x}$ & $\mathrm{x}$ & & & & & & & & & & & & \\
\hline (Sel y Bilgen 2015) & $\mathrm{x}$ & $\mathrm{x}$ & $\mathrm{x}$ & $\mathrm{x}$ & $\mathrm{x}$ & & & & $\mathrm{x}$ & & $\mathrm{x}$ & $\mathrm{x}$ & $\mathrm{x}$ & $\mathrm{x}$ & \\
\hline (Govindan, Soleimani, y Kannan 2015) & $\mathrm{x}$ & $\mathrm{x}$ & $\mathrm{x}$ & & $\mathrm{x}$ & $\mathrm{x}$ & & $\mathrm{x}$ & & & & & & $\mathrm{x}$ & $\mathrm{x}$ \\
\hline (Ho et al. 2015) & $\mathrm{x}$ & $\mathrm{x}$ & $\mathrm{x}$ & $\mathrm{x}$ & $\mathrm{x}$ & $\mathrm{x}$ & $\mathrm{x}$ & $\mathrm{x}$ & $\mathrm{x}$ & & $\mathrm{x}$ & $\mathrm{x}$ & $\mathrm{x}$ & $\mathrm{x}$ & $\mathrm{x}$ \\
\hline (Habib, Lee, y Memon 2016) & & $\mathrm{x}$ & $\mathrm{x}$ & $\mathrm{x}$ & $\mathrm{x}$ & $\mathrm{x}$ & & & & & & & & $\mathrm{x}$ & \\
\hline (Soto-Silva et al. 2016) & & $\mathrm{x}$ & $\mathrm{x}$ & $\mathrm{x}$ & $\mathrm{x}$ & $\mathrm{x}$ & & $\mathrm{x}$ & $\mathrm{x}$ & & & $\mathrm{x}$ & $\mathrm{x}$ & & \\
\hline (Franco y Alfonso-Lizarazo 2017) & & $\mathrm{x}$ & & & $\mathrm{x}$ & & & $\mathrm{x}$ & $\mathrm{x}$ & & & & & $\mathrm{x}$ & \\
\hline
\end{tabular}




\begin{tabular}{|c|c|c|c|c|c|c|c|c|c|c|c|c|c|}
\hline (Zandi Atashbar, Labadie, y Prins 2018) & $\mathrm{x}$ & $\mathrm{x}$ & $\mathrm{x}$ & & $\mathrm{x}$ & $\mathrm{x}$ & $\mathrm{x}$ & $\mathrm{x}$ & $\mathrm{x}$ & $\mathrm{x}$ & & $\mathrm{x}$ & $\mathrm{x}$ \\
\hline (Hamdi et al. 2018) & $\mathrm{x}$ & $\mathrm{x}$ & $\mathrm{x}$ & & $\mathrm{x}$ & $\mathrm{x}$ & & $\mathrm{x}$ & & $\mathrm{x}$ & $\mathrm{x}$ & $\mathrm{x}$ & $\mathrm{x}$ \\
\hline (Kochan y Nowicki 2018) & $\mathrm{x}$ & $\mathrm{x}$ & & $\mathrm{x}$ & $\mathrm{x}$ & & & $\mathrm{x}$ & & $\mathrm{x}$ & $\mathrm{x}$ & $\mathrm{x}$ & $\mathrm{x}$ \\
\hline $\begin{array}{l}\text { (Barbosa-Póvoa, da Silva, y Carvalho } \\
\text { 2018) }\end{array}$ & & $\mathrm{x}$ & & & & & & & & & & & \\
\hline (Mundi et al. 2019) & & & $\mathrm{x}$ & & & $\mathrm{x}$ & & $\mathrm{x}$ & & $\mathrm{x}$ & $\mathrm{x}$ & $\mathrm{x}$ & \\
\hline (Oliveira et al. 2019) & $\mathrm{x}$ & $\mathrm{x}$ & $\mathrm{x}$ & $\mathrm{x}$ & $\mathrm{x}$ & $\mathrm{x}$ & $\mathrm{x}$ & $\mathrm{x}$ & $\mathrm{x}$ & $\mathrm{x}$ & & $\mathrm{x}$ & \\
\hline
\end{tabular}

- A. Modelos Analíticos

- $\quad$ S. Modelos de simulación

1. Programación Lineal (Linear Programming - LP)

2. Programación Lineal Entera (Integer Linear Programming - ILP),

3. Programación Lineal Entera Mixta (Mixed Integer Linear Programming - MILP),

4. Programación no lineal /Entera Mixta / no lineal entera (Non-Linear Programming - NLP) /Mixed integer/Integer non linear programming)

5. Programación cuadrática (Quadratic Programming - QP),

6. Programación dinámica (Dynamic Programming - DP),

7. Programación estocástica (Stochastic Programming - SP),

8. Programación robusta (Robust Programming - RP),

9. Programación por restricciones (Constraint Programming - CP),

10. Programación lineal multi-objetivo / Programación no lineal multi-objetivo/programación no lineal entera mixta multi-objetivo (Multi-Objective Linear Programming - MOLP) / Multi-Objective Non-Linear Problem - MONLP)/Multi-Objective Mixed-Integer Non-Linear Programming - MOMINLP)

11. Enfoques híbridos (Hybrid Approach - HA)

12. Programación difusa (Fuzzy programming - FP)

13. Programación por objetivos (Goal Programming - GP)

\subsection{Métodos de solución}

En la actualidad es de mucha relevancia analizar los métodos de cálculo utilizados para resolver los problemas de optimización, puesto que es un campo de investigación muy amplio y muchos sectores de la industria están haciendo énfasis en su desarrollo. La Tabla 10 describe los algoritmos utilizados en las publicaciones, teniendo en cuenta que la clasificación se hizo en relación con los métodos de resolución propuestos en la Tabla 6 , en la que se especifica la categorización de cada tipo de algoritmo. En general, los investigadores proponen la utilización algoritmos heurísticos y metaheurísticos para la resolución de problemas de gran tamaño (problemas reales), quedando una amplia área por explorar: los llamados algoritmos matheurísticos. Es importante destacar que solamente dos investigaciones Habib et al. (2016) y Zandi Atashbar et al. (2018) contrastaron que métodos o técnicas de solución resuelven los modelos de optimización. 
Tabla 10 Tipos de metodos de resolución

\begin{tabular}{|c|c|c|c|c|}
\hline Autor & Optimizador & Heurístico & Metaheurístico & Matheurístico \\
\hline (Peidro et al. 2009) & $\mathrm{x}$ & $\mathrm{x}$ & $\mathrm{x}$ & \\
\hline (Mula et al. 2010) & & $\mathrm{x}$ & $\mathrm{x}$ & \\
\hline (Stindt y Sahamie 2014) & $\mathrm{x}$ & $\mathrm{x}$ & $\mathrm{x}$ & \\
\hline (Brandenburg et al. 2014) & & $\mathrm{x}$ & $\mathrm{x}$ & \\
\hline (Malviya y Kant 2015) & & $\mathrm{x}$ & & \\
\hline (Sel y Bilgen 2015) & & $\mathrm{x}$ & $\mathrm{x}$ & \\
\hline (Govindan, Soleimani, y Kannan 2015) & $\mathrm{x}$ & $\mathrm{x}$ & $\mathrm{x}$ & \\
\hline (Ho et al. 2015) & & & $\mathrm{x}$ & \\
\hline (Habib, Lee, y Memon 2016) & $\mathrm{x}$ & $\mathrm{x}$ & $\mathrm{x}$ & \\
\hline (Soto-Silva et al. 2016) & & $\mathrm{x}$ & $\mathrm{x}$ & \\
\hline (Franco y Alfonso-Lizarazo 2017) & $\mathrm{x}$ & $\mathrm{x}$ & $\mathrm{x}$ & \\
\hline $\begin{array}{l}\text { (Zandi Atashbar, Labadie, y Prins } \\
\text { 2018) }\end{array}$ & $\mathrm{x}$ & $\mathrm{x}$ & $\mathrm{x}$ & $\mathrm{x}$ \\
\hline (Hamdi et al. 2018) & $\mathrm{x}$ & $\mathrm{x}$ & $\mathrm{x}$ & $\mathrm{x}$ \\
\hline \multicolumn{5}{|l|}{ (Kochan y Nowicki 2018) } \\
\hline $\begin{array}{l}\text { (Barbosa-Póvoa, da Silva, y Carvalho } \\
\text { 2018) }\end{array}$ & & & $\mathrm{x}$ & \\
\hline \multicolumn{5}{|l|}{ (Mundi et al. 2019) } \\
\hline (Oliveira et al. 2019) & $\mathrm{x}$ & $\mathrm{x}$ & $\mathrm{x}$ & \\
\hline
\end{tabular}

\section{Discusión y oportunidades futuras de investigación}

Esta sección discute los resultados de la revisión y las líneas de investigación que proponen las revisiones analizadas, además, en este análisis, se comparan los estudios en relación con la perspectiva o enfoque al que se dirigieron. La tabla 11 detalla un breve resumen de las principales líneas de investigación que proponen las revisiones. Este apartado da respuesta a la PI7. 
Tabla 11 Resumen de futuras líneas de investigación desde las diferentes perspectivas en la cadena de suministro

\begin{tabular}{|c|c|c|}
\hline Perspectiva & Líneas futuras de investigación & Referencias \\
\hline $\begin{array}{l}\text { Cadena de suministro } \\
\text { bajo incertidumbre }\end{array}$ & $\begin{array}{l}\text { - Identificación precisa de fuentes de incertidumbre } \\
\text { Desarrollo de modelos de optimización y técnicas } \\
\text { de solución para abordar la incertidumbre en la de- } \\
\text { manda de productos con falta de homogeneidad } \\
\text { Estudio de la planificación agregada de la produc- } \\
\text { ción para sistemas de fabricación reconfigurable } \\
\text { Estudio de la planificación agregada de la produc- } \\
\text { ción en la industria de procesos en escenarios inci- } \\
\text { ertos o deterministas que consideren un horizonte } \\
\text { rodante } \\
\text { Aplicación a casos reales }\end{array}$ & $\begin{array}{l}\text { (Peidro et al. 2009; Jamal- } \\
\text { nia et al. } 2019 \text { Mundi et al. } \\
\text { 2019;) }\end{array}$ \\
\hline
\end{tabular}

- Desarrollo de modelos que consideren específicamente una industria en particular y no una aplicación general

(Stindt y Sahamie 2014; Govindan, Soleimani, y

Cadena de suministro • • Uso de orientaciones como el enfoque de intervalos de ciclo cerrado (Interval Approaches) y teoría del caos (Chaos Theory)

Kannan 2015; Kazemi, Modak, y Govindan 2018)

- Aplicación de enfoques estocásticos en dos etapas

- Uso de enfoques no deterministas

- $\quad$ Empleo de optimización robusta

\section{Cadena de suministro} verde
- $\quad$ Estudios comparativos intersectoriales de la industria

- $\quad$ Estudios comparativos intercontinentales de la industria

- $\quad$ Aplicación a casos reales
(Tseng et al. 2019)

- Análisis de aspectos sociales, y su cuantificación integración con aspectos económicos y ambientales

- Gestión de riesgos ambientales

- $\quad$ Estudio de enfoques híbridos, en los que se combi-

Cadena de suministro nen metaheurísticas, matheurísticas u otros tipos de sostenible métodos más eficientes

(Brandenburg et al. 2014; Barbosa-Póvoa, da Silva, y Carvalho 2018)

- Aplicación a sectores como la industria farmacéutica, la confección, la energía y el transporte

- Análisis de sostenibilidad en ámbitos como el transporte y en el almacenamiento 


\begin{tabular}{|c|c|c|c|}
\hline $\begin{array}{l}\text { Riesgo de la cadena } \\
\text { de suministro }\end{array}$ & $\begin{array}{l}\cdot \\
\cdot \\
\cdot \\
\cdot \\
\cdot \\
\cdot \\
\cdot \\
\cdot \\
\\
\end{array}$ & $\begin{array}{l}\text { Análisis de riesgos infraestructurales, el riesgo fi- } \\
\text { nanciero y de la información } \\
\text { Investigación del riesgo de producción y de los pro- } \\
\text { cesos } \\
\text { Medición de la relación entre los factores de riesgo } \\
\text { y el tipo de riesgo, relacionándolo con la probabili- } \\
\text { dad de ocurrencia } \\
\text { Validación de los métodos de gestión del riesgo } \\
\text { para evaluar y seleccionar las mejores estrategias } \\
\text { de mitigación de riesgos, examinando entre las es- } \\
\text { trategias individuales e integradas } \\
\text { Análisis de las cadenas de suministro de servicios } \\
\text { como banca, seguros, medicina } \\
\text { Uso los métodos de simulación para reproducir la } \\
\text { dinámica del riesgo y los impactos del riesgo } \\
\text { Desarrollo de modelos para la gestión del riesgo } \\
\text { como el de la colonia de hormigas (Ant Colony) o } \\
\text { el método de intersección de límites normales (Nor- } \\
\text { mal Boundary Intersection) } \\
\text { Uso de técnicas de programación estocástica o pro- } \\
\text { gramación difusa } \\
\text { Hibridación entre enfoques de programación } \\
\text { matemática junto con técnicas de inteligencia artifi- } \\
\text { cial para la ayuda a la tomar decisiones automatiza- } \\
\text { da, basada en la predicción y el aprendizaje }\end{array}$ & $\begin{array}{l}\text { (Hamdi et al. 2018; Bary- } \\
\text { annis et al. 2018; Oliveira } \\
\text { et al. 2019) }\end{array}$ \\
\hline $\begin{array}{l}\text { Resiliencia en la cade- } \\
\text { na de suministro }\end{array}$ & $\begin{array}{l}\cdot \\
\cdot \\
\cdot \\
\cdot \\
\cdot\end{array}$ & $\begin{array}{l}\text { Análisis de la relación entre la cadena de suministro } \\
\text { verde, la resiliencia y el análisis de la resiliencia en } \\
\text { la industria } 4.0 \\
\text { Uso de métodos analíticos como AHP, ANP, TOP- } \\
\text { SIS, ELECTRE, VIKOR } \\
\text { Explorar los métodos de toma de decisiones } \\
\text { multi-criterio (Multi-Criteria Decision-Making - } \\
\text { MCDM) } \\
\text { Desarrollo de técnicas de programación estocástica } \\
\text { de dos etapas con múltiples objetivos y uso de la } \\
\text { programación por restricciones (Constraint Pro- } \\
\text { gramming) }\end{array}$ & $\begin{array}{l}\text { (Kochan y Nowicki 2018; } \\
\text { Hosseini, Ivanov, y Dolgui } \\
\text { 2019) }\end{array}$ \\
\hline
\end{tabular}

La Tabla 12 especifica algunos sectores que proponen interesantes líneas de investigación en la gestión de la cadena de suministro. 
Tabla 12 Resumen de futuras líneas de investigación desde la perspectiva de la gestión de la cadena de suministro considerando varios sectores

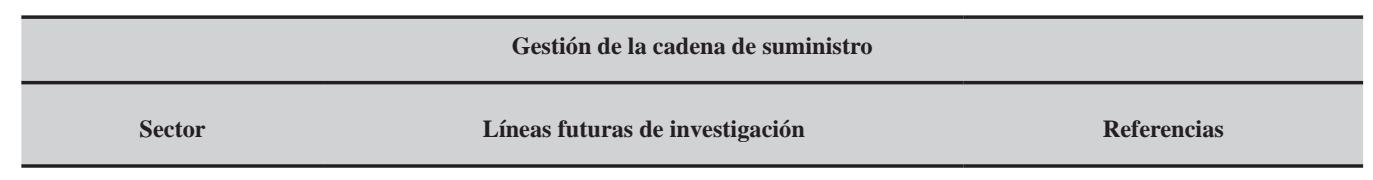

- Planteamiento de modelos realistas que tengan en cuenta aspectos de la vida real como las políticas, prácticas y procedimientos para el manejo de desastres y el procesamiento de escombros

- $\quad$ Estudio de la gestión de la cadena de suministro humanitaria adoptando políticas de la cadena de suministro verde

\begin{tabular}{|c|c|c|c|}
\hline Sector: lácteo & • & $\begin{array}{l}\text { Estudio de modelos que consideren temas } \\
\text { como la trazabilidad y aspectos relacionados } \\
\text { a la seguridad alimentaria, y aspectos como la } \\
\text { minimización de residuos }\end{array}$ & (Sel y Bilgen 2015) \\
\hline Sector: fruta fresca & • & $\begin{array}{l}\text { Diseño de enfoques holísticos para el diseño y } \\
\text { la gestión de las cadenas de suministro agríco- } \\
\text { la y de productos perecederos } \\
\text { Estudio la producción de fruta orgánica } \\
\text { Estudio de técnicas integradas de simulación y } \\
\text { optimización, considerando la evolución de un } \\
\text { enfoque mono criterio a uno multicriterio }\end{array}$ & (Soto-Silva et al. 2016) \\
\hline Sector: farmacéutico & • & $\begin{array}{l}\text { Estudio de métodos cuantitativos para el área } \\
\text { de la atención médica o estudios de la logística } \\
\text { en los hospitales } \\
\text { Uso de técnicas combinadas o hibridas para } \\
\text { aproximar el funcionamiento de la cadena de } \\
\text { suministro farmacéutica a los casos de la vida } \\
\text { real } \\
\text { Empleo de técnicas como la programación di- } \\
\text { fusa o programación robusta }\end{array}$ & (Franco y Alfonso-Lizarazo 2017) \\
\hline Sector: biomasa & • & $\begin{array}{l}\text { Uso de técnicas de descomposición o relaja- } \\
\text { ción, técnicas metaheurísticas o algoritmos } \\
\text { evolutivos y métodos de optimización basados } \\
\text { en simulación }\end{array}$ & $\begin{array}{l}\text { (Zandi Atashbar, Labadie, y Prins } \\
\text { 2018) }\end{array}$ \\
\hline
\end{tabular}

A continuación, se presenta un análisis detallado sobre las líneas futuras de investigación desde las diferentes perspectivas en la cadena de suministro y en la gestión de la cadena de suministro desde la perspectiva sectorial.

\subsection{Cadena de suministro bajo incertidumbre}

Las investigaciones sobre la cadena de suministro bajo incertidumbre convergen en que aún no se han identificado todo el conjunto de fuentes de incertidumbre potenciales, independientemente del campo de estudio. De igual forma las revisiones analizadas señalan que faltan estudios aplicados a casos reales.
Con respecto a lo mencionado, dos revisiones estudian la cadena de suministro desde la perspectiva de incertidumbre (Peidro et al. 2009; Mundi et al. 2019). Entre ambos estudios hay 10 años de diferencia, no obstante, los dos coinciden que trabajos futuros deben desarrollar modelos de programación difusa para manejar datos imprecisos y/o no disponibles. Es importante señalar que Mundi et al. (2019) utilizaron el trabajo de (Peidro et al. 2009) como base para su investigación, añadiendo la característica de falta de homogeneidad en los productos. Además, Peidro et al. (2009), sugieren otras líneas de investigación como el desarrollo de nuevos modelos híbridos mediante la combinación de modelos basados tanto en la teoría de conjuntos difusos como en la simulación, que puedan considerar enfoques de control predicti- 
vo. Asimismo, resaltan la falta de aplicación de los enfoques de modelado a casos reales. Los autores hacen hincapié en la utilización de modelos basados en inteligencia artificial, y en el análisis de los mecanismos de gestión de la incertidumbre desde una perspectiva cuantitativa. Por otra parte, Mundi et al. (2019) sugieren el desarrollo de modelos de optimización y técnicas de solución para abordar la incertidumbre en la demanda de productos con falta de homogeneidad.

La perspectiva de cadena de suministro bajo incertidumbre es un tema ampliamente estudiado, un ejemplo de esto es la revisión presentada por Jamalnia et al.(2019), los cuales analizan la planificación agregada de la producción bajo incertidumbre, concluyendo que los modelos de programación difusa son los más estudiados seguidos de los programación estocástica. De esta forma podemos concluir las líneas de investigación futuras identificadas en el estudio de Peidro et al. (2009) han sido abordadas por una gran cantidad de autores y mantienen su vigencia. Sin embargo, en esta área de investigación aún quedan brechas por cubrir como las mencionadas por el estudio de Mundi et al. (2019) y las presentadas por Jamalnia et al.(2019), este último, menciona en su publicación que existen muy pocos estudios sobre la planificación agregada de la producción para sistemas de fabricación reconfigurable, y en la industria de procesos (refinerías de petróleo, la fabricación de bebidas y los procesos químicos que operan procesos de producción continuos e ininterrumpidos) en escenarios inciertos o deterministas que consideren un horizonte rodante.

\subsection{Cadena de suministro de ciclo cerrado}

La cadena de suministro ciclo cerrado presenta un área con grandes oportunidades de investigación. Últimas publicaciones, como la de Kazemi et al. (2018) presenta que la mayoría de estudios de la cadena de suministro de ciclo cerrado se centran únicamente en las consecuencias económicas directas, como los costos operativos y las ganancias de las ventas. No obstante, la cadena de suministro de ciclo cerrado busca mitigar los daños y riesgos ambientales y mejorar la vida social, por lo que modelar en conjunto los parámetros ambientales, sociales y económicos puede ser una importante área de investigación.

Todas estas observaciones se relacionan también con estudios anteriores como el de Govindan et al. (2015), en el que además sugiere el estudio de enfoques no deterministas, como el enfoque de intervalos (Interval Approaches) y teoría del caos (Chaos Theory), para tratar con la incertidumbre, y también consideran que se pueden integrar diferentes enfoques no deterministas. Además, contemplan la utilización de enfoques estocásticos en dos etapas y la optimización robusta, para tratar con la incertidumbre que caracteriza a las situaciones reales. Igualmente platean el uso programación no lineal y optimización convexa, para tratar problemas reales. Govindan et al. (2015) lanzan la propuesta de investigar la aplicación de algoritmos de aproximación o algoritmos híbridos, dado que pueden presentar una solución aceptable al resolver problemas complejos con menor tiempo de computación. Finalmente, proponen el análisis de métodos de pronóstico de la incertidumbre, ya que sus hallazgos fueron en su mayoría conceptuales.

En esta misma línea, Stindt y Sahamie (2014) plantean el desarrollo de modelos y métodos de solución cuantitativos específicos para cada sector de la industria de procesos o manufactura, en los que se tengan en cuenta las circunstancias de cada sector de forma individual ya que se han analizado parámetros generales de esta industria en los modelos.

Podemos destacar que aún existen oportunidades de investigación a explotar en este campo, lo que ha permitido concluir que la cadena de suministro de ciclo cerrado está teniendo un avance muy rápido respecto a las publicaciones. Un ejemplo de esto, es el estudio de Kazemi et al. (2018), en el que se analizaron 94 estudios entre 2000 al 2017, actualizando el estudio de Govindan, Soleimani, y Kannan (2015). Ambas revisiones coinciden con Stindt y Sahamie (2014), identificando una falta de modelos desarrollados específicamente para tipos de industrias particulares. Los autores complementan sus conclusiones haciendo hincapié en que los modelos que pueden funcionar en un sector específico pueden no hacerlo en otro distinto. A su vez, consideran la utilización de métodos como teoría del caos o teoría de conjuntos aproximados y la optimización robusta para tratar la incertidumbre.

\subsection{Cadena de suministro verde}

Las industrias cada vez más se ven comprometidas a incorporar en concepto "verde" en la cadena de suministro, esto significa incorporar el concepto ambiental en todas las etapas del producto, desde el diseño hasta el final de su vida útil, y que en esta etapa final el producto pueda reciclarse, reutilizarse o recuperarse (Gandhi y Vasudevan 2019).

Uno de los principales hallazgos encontrados, en el contexto de la cadena de suministro verde, concluye que la mayoría de los autores proponen métodos de simulación para modelar los problemas, mientras que los modelos matemáticos se utilizan de forma limitada (Malviya y Kant 2015). En contraste con este hallazgo, publicaciones recientes como la de Tseng et al. (2019) revelan que hay un incremento de artículos que plantean modelos matemáticos para la optimización de la toma de decisiones en la gestión de la cadena de suministro verde.

En definitiva la gestión de la cadena de suministro verde es una área a la que, según Tseng et al. (2019), se está prestando mucho interés debido a la presión que tienen las empresas para mejorar el desempeño ambiental por parte de los gobiernos. Si bien las líneas de investigación futuras que se plantearon en el 2015 hasta el 2019 ya han sido cubiertas, según Tseng et al. (2019) aún quedan áreas por 
explorar. Vale la pena mencionar la línea de investigación que sugiere la realización de estudios comparativos intersectoriales de la industria y estudios comparativos intercontinentales, puesto que estas investigaciones son escasas en la literatura. Tseng et al. (2019) también enfatiza en la falta de estudios que presenten datos de la vida real.

\subsection{Cadena de suministro sostenible}

La gestión sostenible de la cadena de suministro es un enfoque que empuja a las empresas a mejorar desde la perspectiva de la sostenibilidad, es decir que las empresas se ocupen de integrar los objetivos económicos, sociales y ambientales en los procesos de la cadena de suministro (Koberg y Longoni 2019).

En relación a este tema, Brandenburg et al. (2014) estudian los métodos cuantitativos utilizados en la gestión sostenible de la cadena de suministro, describiendo que los modelos que tratan este tema están principalmente relacionados a las industrias de manufactura, como la industria automotriz y textil; siendo pocos los estudios enmarcados en los sectores de productos químicos y de petróleo. Así mismo, los autores identifican la falta de atención a la gestión de riesgos ambientales y la incorporación aspectos sociales en los modelos planteados. Finalmente, Brandenburg et al. (2014) propone que se analice la sostenibilidad en ámbitos como el transporte y en el almacenamiento, destacando además que tanto los algoritmos evolutivos como las técnicas como la programación dinámica y los métodos de búsqueda local no han sido estudiados en profundidad.

Una revisión más actual de este tema es la presentada por Barbosa-Povoa et al. (2018), identificando que los métodos de programación matemática son los más utilizados, seguido por las técnicas de simulación. En cuanto a la combinación de técnicas, la combinación más abordada es la programación matemática con técnicas de simulación, seguido por métodos analíticos y metaheurísticas. Barbosa-Povoa et al. (2018) coincide con Brandenburg et al. (2014) en que son pocos los modelos que han abordado aspectos sociales.

Barbosa-Povoa et al. (2018) destaca que en el 2015 las técnicas de optimización alcanzaron un pico en las publicaciones y que hubo un gran crecimiento de las aplicaciones industriales reales en las que utilizan técnicas de optimización en sus publicaciones y concuerda con Brandenburg et al. (2014) en que el sector de la manufactura (automotriz, textil etc.) es significativamente estudiado, sin embargo, en los cuatro años de diferencia que existen entre ambas revisiones, las publicaciones que proponen modelos de optimización aplicados a la industria de procesos se han incrementado ocupando el primer lugar según mencionan Barbosa-Povoa et al. (2018). Además concuerda con Brandenburg et al. (2014) en que se deben estudiar aspectos sociales y cuantificarlos, proponiendo para ello que se estudien los enfoques híbridos, en los que se combinen metaheurística, matheurísticas u otros tipos de métodos más eficientes. En cuanto a los enfoques industriales, los sectores como la industria farmacéutica, la confección, la energía y el transporte requieren un estudio adicional.

En definitiva la gestión sostenible de la cadena de suministro es un área que presenta importantes líneas de investigación como las mencionadas por Barbosa-Povoa et al. (2018), que en gran parte de su investigación coincide y amplia la investigación de Brandenburg et al. (2014). En general estas líneas, se relacionan a que existen sectores de la industria que necesitan ser estudiados y que sus modelos deben combinar aspectos económicos, sociales y ambientales, mediante el uso de técnicas robustas. De igual forma es importante resaltar lo mencionado por Barbosa-Povoa et al. (2018), destacando la existencia de una brecha de investigación en temas como la cadena de suministro de ciclo cerrado y la gestión inversa de la cadena de suministro que traten temas económicos, sociales y ambientales en los procesos de la cadena de suministro.

Respecto a la gestión inversa de la cadena de suministro, se concluye que es un área que está en crecimiento, y que presenta significativas áreas de investigación, sin embargo, en la búsqueda realizada en el presente artículo no se han encontrado estudios que analicen en detalle este tema y respondan a nuestras preguntas de investigación.

\subsection{Riesgo de la cadena de suministro}

Según Baryannis et al. (2018) el interés de los investigadores por la gestión del riesgo en la cadena de suministro ha incrementado significativamente las últimas dos décadas, por tres razones: (i) la adopción de filosofías como "lean manufacturing” o "just-in-time" en los procesos de fabricación dejando un margen reducido para el error y el cambio; (ii) la exposición a más riesgos ya que las empresas son cada vez más globales y menos verticales; y (iii) la probabilidad de ocurrencia de desastres o eventos que perturban la cadena de suministro global como desastres naturales, escasez, por ejemplo: materia prima etc.

En este contexto, estudios como el de Ho et al. (2015) recomiendan que se analicen los riesgos infraestructurales como el transporte, el riesgo financiero y de la información, así como examinar el riesgo de fabricación o producción y el de los procesos. De igual forma proponen que se mida la relación entre los factores de riesgo y el tipo de riesgo, relacionándolo con la probabilidad de ocurrencia. Ho et al. (2015) enfatizan la validación de marcos conceptuales y métodos de gestión del riesgo para evaluar y seleccionar las mejores estrategias de mitigación de riesgos, examinando entre las estrategias individuales e integradas, de esta forma medir en función su eficiencia y eficacia. Así mismo, los autores proponen que se analicen las cadenas de suministro de servicios como banca, seguros, medicina, etc. 
Por otra parte, Hamdi et al. (2018) estudian la selección de proveedores bajo el enfoque de la gestión del riesgo de la cadena de suministro, señalando que la investigación cuantitativa es predominante en este campo, especialmente en lo que respecta a la formulación matemática. También son preponderantes los enfoques AHP para la selección de proveedores, y los enfoques de optimización estocástica que se usan para modelar las fluctuaciones y los cambios entrantes en la cadena de suministro. Estos autores proponen que se indague en la combinación de técnicas, con el objetivo de minimizar el riesgo del proveedor, es decir que se analice el caso de la interrupción simultánea de proveedores locales, globales y semi-globales.

De igual modo Oliveira et al. (2019) abordan la problemática de plantear estrategias para mitigar el riesgo, concluyendo que no hay una alineación entre la respuesta al riesgo y los enfoques de solución del riesgo, ya que las estrategias de respuesta al riesgo deben ser coherentes con las soluciones para mitigar el riesgo. Este estudio analiza la aplicación de herramientas de simulación, modelos de optimización y sistemas de medición de rendimiento para la gestión del riesgo de la cadena de suministro. Respecto a la simulación y optimización, los autores proponen el uso de los métodos de simulación para reproducir la dinámica del riesgo y los impactos del riesgo, y el uso de modelos para la gestión del riesgo como el de la colonia de hormigas (Ant Colony) y el método de intersección de límites normales (Normal Boundary Intersection). Dado que no se encontraron estos métodos en los artículos revisados, Oliveira et al. (2019) sugieren que se mejore la perspectiva de la optimización basada en la simulación, además propone que se desarrollen medidas de riesgo basadas en Seis Sigma para evaluar los impactos críticos del riesgo en el rendimiento de la cadena de suministro.

Como resultado de estas investigaciones podemos concluir que el área de gestión del riesgo de la cadena de suministro presenta varias líneas de investigación interesantes, como las plateadas por Baryannis et al. (2018), que hicieron una revisión de 276 artículos sobre la gestión del riesgo en la cadena de suministro y su relación con técnicas de inteligencia artificial. Del total de artículos, analizados por Baryannis et al. (2018), el $84 \%$ se centran en la respuesta al riesgo en modelos que evitan o mitigan los efectos del riesgo y la incertidumbre, el $4 \%$ combinan la respuesta con alguna forma de evaluación del riesgo, el 4\% combinan la evaluación de los riesgos con la identificación de los riesgos, el 3\% abordan individualmente la identificación de riesgos, el 2\% evalúan los riesgos, y el 3\% usa enfoques holísticos para la identificación, evaluación y respuesta al riesgo. También describen que la mayoría de las técnicas utilizadas son la de programación matemática coincidiendo con las conclusiones que se derivan del estudio de Hamdi et al. (2018). En este sentido, ambas revisiones concluyen que los investigadores se han decantado por desarrollar técnicas de programación matemática, siendo menos aplicadas las técnicas de Inteli- gencia Artificial como modelos basados en agentes y redes, razonamiento automático, aprendizaje automático y analítica de Big Data. Los dos trabajos identifican que técnicas como la programación estocástica o programación difusa son poco utilizadas. Finalmente mencionan que el campo de la Inteligencia Artificial para la gestión proactiva y predictiva del riesgo en la cadena de suministro es una gran oportunidad de investigación, y proponen que se investigue una hibridación entre enfoques de programación matemática junto con una técnica de inteligencia artificial para que ayude a tomar decisiones de forma automatizada basada en la predicción y el aprendizaje.

\subsection{Resiliencia en la cadena de suministro}

Un tema relacionado con la gestión del riesgo en la cadena de suministro es la resiliencia de la cadena de suministro. En este contexto, el estudio llevado a cabo por Kochan y Nowicki (2018) menciona que no existe una definición unánime de la resiliencia de la cadena de suministro. Sin embargo según Burnard et al. (2019) la resiliencia ofrece a las empresas una capacidad dinámica de anticiparse, responder y adaptarse a los riesgos y amenazas,

Respecto a la resiliencia en la cadena de suministro, uno de los estudios más recientes es el de Hosseini et al. (2019), donde se plantean interesantes áreas de investigación como el uso de métodos analíticos, incluyendo AHP, ANP, TOPSIS, ELECTRE, VIKOR, que no han sido analizados en el contexto de la resiliencia de la cadena de suministro, también recomienda que las líneas futuras de investigación se centren en explorar los métodos de toma de decisiones multi-criterio (Multi-Criteria Decision-Making, MCDM) concluyendo que dichos métodos son aplicables para evaluar las redes de la cadena de suministro, en relación con la resiliencia, criterios ecológicos y organizativos.

Otro aspecto propuesto por Hosseini et al. (2019) es centrarse en la programación estocástica de dos etapas con múltiples objetivos, ya que la resiliencia de la cadena suministro esta intrínsicamente relacionada con aspectos estocásticos como la perdida de la capacidad, la recuperación de la capacidad y la restauración de los recursos, así como del tiempo esperado de recuperación. De igual forma proponen que se planteen modelos de optimización robusta que, según su investigación, aún no ha sido abordada. Los autores también proponen el uso de la programación por restricciones (Constraint Programming) en el contexto de la resiliencia de la cadena de suministro, de forma general plantean el estudio de la relación entre la cadena de suministro verde, la resiliencia y el análisis de la resiliencia en la industria 4.0. En definitiva, las áreas de investigación futuras planteadas por estas investigaciones recientes pueden dar varias posibilidades de estudios para los investigadores. 


\subsection{Gestión de la cadena de suministro.}

La gestión de la cadena de suministro es un área muy amplia que es aplicable a diferentes sectores y que tiene diferentes enfoques, en este apartado hemos detallado algunos sectores que proponen interesantes líneas de investigación.

\subsubsection{Sector: humanitario}

Una nueva área que está empezando a surgir es la de la cadena de suministro humanitaria. Según Habib et al. (2016) dicho sector tiene una gran área de investigación inexplorada ya que la mayoría de los artículos sobre estos temas surgieron a partir del 2004, con el terremoto del océano Índico, además confirman que la gestión de la cadena de suministro en su ámbito comercial con enfoque en la sostenibilidad ha tenido mucha investigación, mientras que en la gestión de la cadena de suministro humanitaria aún existen pocos estudios. En la revisión llevada a cabo por Habib et al. (2016) se concluye que la formulación de los modelos, planteados en el ámbito humanitario, son pocos realistas y de difícil aplicación. Los autores proponen que la gestión de la cadena de suministro humanitaria adopte políticas de la cadena de suministro verde y que cuando se vayan a generar nuevos modelos se tengan en cuenta varios aspectos de la vida real como las políticas, prácticas y procedimientos para el manejo de desastres y el procesamiento de escombros.

\subsubsection{Sector: lácteo}

Sel y Bilgen (2015) llevan a cabo la revisión y discusión de modelos cuantitativos para la gestión de la cadena de suministro dentro de la industria láctea. La mayoría de los artículos analizados por los autores utilizan modelos de programación entera mixta, por ello proponen que en dichos modelos se tenga en cuenta aspectos clave para el sector como la trazabilidad y la seguridad alimentaria. Otras líneas de investigación futuras se definen hacia temas de minimización de residuos, y la programación por restricciones (Constraint Programming) en la planificación de la producción y la distribución, ya que hasta el momento no han sido abordados. En este mismo sentido Sel y Bilgen (2015) mencionan que existe pocas publicaciones que utilicen métodos heurísticos, metaheurísticos o hibridaciones de estos. También propone la utilización de heurísticas basadas en modelos de programación entera mixta relajada, y enfoques de horizonte rodante. Los autores consideran que hay pocas combinaciones de modelos programación matemática con métodos de solución aproximada, en el contexto de cadena de suministro láctea. Otras sugerencias propuestas para desarrollar en futuras investigaciones es el desarrollo de modelos de programación estocásticos o multiparamétricos.

\subsubsection{Sector: fruta fresca}

Soto-Silva et al. (2016) realizan una revisión de modelos de investigación operativa aplicados a la cadena de suministro de fruta fresca. Los autores describen la falta de enfoques holísticos para el diseño y la gestión de las cadenas de suministro agrícola, ocurriendo lo mismo en el caso de productos perecederos. En este campo se han abordado temáticas como la programación optima de las cosechas y la simulación logística de eventos discretos. Se está dando mucha importancia a la sostenibilidad y a los problemas ambientales, así como a temas de seguridad alimentaria. Soto-Silva et al. (2016) concluyen que los problemas encontrados en la revisión están relacionados con el transporte, la planificación y la asignación en las etapas de producción y distribución, siendo los modelos de programación lineal, programación lineal entera o entera mixta los más utilizados. En esta área, según se menciona en la revisión, los artículos analizados son de casos reales. En este sentido, los autores proponen que se analice la producción de fruta orgánica, y en los temas de optimización que se usen técnicas integradas de simulación y optimización y que se pase de un enfoque mono criterio a uno multicriterio.

\subsubsection{Sector: farmacéutico}

Franco y Alfonso-Lizarazo (2017) analizan la cadena de suministro farmacéutica mencionando que no es un tema exhaustivamente estudiado, principalmente en los métodos cuantitativos planteados en el área de la atención médica o estudios de la logística en los hospitales. Los autores describen que la mayoría de los estudios analizados, utilizan una forma estocástica de incertidumbre en algún escalón de la cadena de suministro, y solo el $20 \%$ corresponden a modelos de optimización de la cadena de suministro, el $52 \%$ pertenecen a diseño de redes y el $28 \%$ a problemas inventario. De los modelos planteados el $92 \%$ utilizan datos de la demanda de forma estocástica y el resto de forma determinista. Además, Franco y Alfonso-Lizarazo (2017) destacan que pocos artículos utilizan programación difusa o programación robusta, por lo que, proponen que se utilicen técnicas combinadas para aproximar el funcionamiento de la cadena de suministro farmacéutica a los casos de la vida real.

\subsubsection{Sector: biomasa}

Zandi Atashbar et al. (2018) comentan que la mayoría de las investigaciones que estudian la gestión de la cadena de suministro en el sector de la biomasa proceden de las áreas de agricultura o química. Los investigadores de dichas áreas son expertos en estos temas, pero, hay muy pocos especialistas en el área de investigación de operaciones 
que estén involucrados en el sector de la biomasa, siendo un ámbito importante de investigación y de aporte, por el conocimiento adquirido de otras áreas del sector industrial. Zandi Atash-bar et al. (2018) también identifican que las técnicas de descomposición o relajación son poco utilizadas al igual que las técnicas metaheurísticas o algoritmos evolutivos, lanzando la propuesta de utilizar métodos de optimización basados en simulación.

En esta sección se han analizado artículos de revisión que tratan el concepto de gestión de la cadena de suministro, considerando diferentes sectores relevantes. No obstante, otros autores proponen revisiones en el contexto de gestión de la cadena de suministro, indistintamente del sector. Así, Mula et al. (2010) estudian a la cadena de suministro independientemente del sector, identificando la falta de propuestas que optimicen simultáneamente la planificación de la producción y el transporte, y proponiendo que en la generación de estos modelos se deben tener en cuenta características del transporte como: las restricciones ambientales y consideraciones de las formas de transporte. Además, los autores proponen la integración de los niveles de decisión táctica u operativa y una estructura de planificación colaborativa para gestionar la información compartida con los socios de la cadena de suministro. Así mismo, Mula et al. (2010) reconocen como línea de investigación futura la integración de las herramientas de optimización: simulación, optimización difusa, sistemas multi-agente y algoritmos evolutivos.

En definitiva, el número de estudios analizando modelos y algoritmos para la optimización de planes de aprovisionamiento, producción y distribución de la cadena de suministro sigue aumentando. No obstante, a pesar de esto, son pocos los trabajos que se dediquen claramente a la revisión de áreas específicas, y en general son pocos los desarrollos con aplicaciones prácticas reales sobre el área de investigación abordada en este artículo.

\section{Conclusiones}

Este estudio realiza una revisión los modelos cuantitativos y los métodos de investigación operativa para resolver problemas de optimización relacionados al aprovisionamiento, producción y distribución en la cadena de suministro, buscando de esta forma obtener una mejor compresión de las áreas de la cadena suministro que actualmente son tendencia en la investigación. Teniendo cuenta las diferentes revisiones llevadas a cabo en la literatura, a nuestro mejor entender, no existe una revisión centrada en el análisis de dichas revisiones, lo cual crea una apremiante necesidad, pues el crecimiento de las investigaciones hace que sea difícil para los investigadores tener claridad sobre el área de la cadena de suministro que se quiere investigar y, de igual forma, ocurre con las herramientas que se están utilizando para solucionar los problemas optimización presentes en la cadena de suministro.
En consecuencia, este estudio proporciona un análisis exhaustivo del estado actual de las investigaciones, considerando que estos estudios se centraron específicamente en un área en particular, presentando un análisis del contenido que comprende la diferenciación de categorías que responden a las preguntas de investigación planteadas. En particular se identificaron, consolidaron y sintetizaron los procesos de la cadena de suministro a través de modelo SCOR y los métodos y modelos planteados en las revisiones para resolver problemas de optimización, explorando temas claves, tendencias de investigación y desarrollos que se están realizando en las diferentes áreas de la cadena de suministro.

Acorde con lo mencionado y respondiendo a la PI7, del análisis realizado se observa que la mayoría de las revisiones coinciden en que son muy pocos los estudios que emplean datos reales, y en la falta de estudios comparativos entre los diferentes sectores de la industria, pues el éxito en un área se puede extrapolar a otras áreas, por lo que sería interesante analizar este tema. Así mismo se han hallado muchos modelos y marcos conceptuales que generan una base teórica importante, sin embargo, se concluye que es importarte que se validen estos modelos y se llegue a un consenso de las conceptualizaciones, generando de esta forma una visión integral.

Otro hallazgo importante es que enfoques como la sostenibilidad y los enfoques ambientales cada vez son más considerados al plantear los modelos. Sin embargo, queda un área grande por explorar pues, si bien ha crecido, las revisiones coinciden en que las variables a las que se sujetan los modelos tienen que incorporar las nuevas restricciones que exigen los gobiernos y los mercados actuales. Además, los métodos de optimización robusta y programación difusa aún siguen siendo una oportunidad de investigación en los enfoques de modelado. De igual forma, en los niveles de decisión sigue siendo importante analizar su combinación, y temas como el horizonte rodante han sido utilizados en pocos artículos y en las revisiones fueron muy pocos los que mencionaron brevemente el tema, siendo un área significativa por explorar.

Finalmente, es de gran importancia resaltar que hay un número creciente de artículos que presentan modelos matemáticos y analíticos, detectando un área en la que queda investigación por realizar: los modelos de simulación y la combinación con otros métodos. Muchos de los modelos usan métodos heurísticos o metaheurísticos para su resolución, quedando por explorar el área de la hibridación de estos métodos o la utilización de métodos matheurísticos. Aunque varios estudios concuerdan que los modelos los modelos matemáticos más utilizados son los de programación lineal/entera/entera mixta sigue siendo interesante analizar métodos matheurísticos debido a la dimensión de los problemas reales, con conjuntos de datos grandes, que no son abordables en la actualidad por 
los solucionadores comerciales. Esta pues, es un área de investigación notable, la cual permite la transformación de métodos de programación matemática hacia modelos que puedan resolverse con algoritmos matheurísticos.

\section{Referencias}

Andres, Beatriz, Raquel Sanchis, Jaques Lamothe, Leila Saari, y Frederic Hauser. 2017. «Integrated production-distribution planning optimization models: A review in collaborative networks context». International Journal of Production Management and Engineering 5 (1): 31. https://doi.org/10.4995/ijpme.2017.6807.

Andres, Beatriz, Raquel Sanchis, Raul Poler, y Leila Saari. 2017. «A Proposal of Standardised Data Model for Cloud Manufacturing Collaborative Networks». En Collaboration in a Data-Rich World, editado por Luis M Camarinha-Matos, Hamideh Afsarmanesh, y Rosanna Fornasiero, 77-85. Cham: Springer International Publishing.

Barbosa-Povoa, Ana Paula, Cada da Silva, y Ana Carvalho. 2018. «Opportunities and challenges in sustainable supply chain: An operations research perspective». European Journal of Operational Research 268 (2): 399-431. https://doi.org/10.1016/j.ejor.2017.10.036.

Barbosa-Póvoa, Ana Paula, Cátia da Silva, y Ana Carvalho. 2018. «Opportunities and challenges in sustainable supply chain: An operations research perspective». European Journal of Operational Research 268 (2): 399-431. https://doi.org/10.1016/j.ejor.2017.10.036.

Baryannis, George, Sahar Validi, Samir Dani, y Grigoris Antoniou. 2018. «Supply chain risk management and artificial intelligence: state of the art and future research directions». International Journal of Production Research 0 (0): 1-24. https://doi.org/10.1080/00207543.2018.153 0476.

Brandenburg, Marcus, Kannan Govindan, Joseph Sarkis, y Stefan Seuring. 2014. «Quantitative models for sustainable supply chain management: Developments and directions». European Journal of Operational Research 233 (2): 299-312. https://doi.org/10.1016/j.ejor.2013.09.032.

Burnard, Kevin John, y Ran Bhamra. 2019. «Challenges for organisational resilience». Continuity \& Resilience Review 1 (1): 17-25. https://doi.org/10.1108/crr-01-20190008 .

C2NET. 2016. «Taxonomy of optimisation and simulation solutions for Manufacturing and Logistics Processes».
Fahimnia, Behnam, Christopher S. Tang, Hoda Davarzani, y Joseph Sarkis. 2015. «Quantitative models for managing supply chain risks: A review». European Journal of Operational Research 247 (1): 1-15. https://doi. org/10.1016/j.ejor.2015.04.034.

Fathian, Mohammad, Javid Jouzdani, Mehdi Heydari, y Ahmad Makui. 2018. "Location and transportation planning in supply chains under uncertainty and congestion by using an improved electromagnetism-like algorithm». Journal of Intelligent Manufacturing 29 (7): 1447-64. https://doi.org/10.1007/s10845-015-1191-9.

Franco, Carlos, y Edgar Alfonso-Lizarazo. 2017. «A Structured Review of Quantitative Models of the Pharmaceutical Supply Chain». Complexity 2017: 1-13. https://doi. org/10.1155/2017/5297406.

Gandhi, Meeta, y Hari Vasudevan. 2019. «Green Supply Chain Management Practices and Its Impact on Business Performance». En Proceedings of International Conference on Intelligent Manufacturing and Automation, editado por Hari Vasudevan, Vijaya Kumar N Kottur, y Amool A Raina, 601-11. Singapore: Springer Singapore.

Govindan, Kannan, Hamed Soleimani, y Devika Kannan. 2015. «Reverse logistics and closed-loop supply chain: A comprehensive review to explore the future». European Journal of Operational Research 240 (3): 603-26. https://doi.org/10.1016/j.ejor.2014.07.012.

Habib, Muhammad Salman, Young Hae Lee, y Muhammad Saad Memon. 2016. «Mathematical Models in Humanitarian Supply Chain Management: A Systematic Literature Review». Mathematical Problems in Engineering 2016: 1-20. https://doi.org/10.1155/2016/3212095.

Hamdi, Faiza, Ahmed Ghorbel, Faouzi Masmoudi, y Lionel Dupont. 2018. «Optimization of a supply portfolio in the context of supply chain risk management: literature review». Journal of Intelligent Manufacturing 29 (4): 76388. https://doi.org/10.1007/s10845-015-1128-3.

Handfield, Robert B, y Ernest L Nichols. 1999. Introduction to supply chain management. Upper Saddle River: Prentice Hall.

Ho, William, Tian Zheng, Hakan Yildiz, y Srinivas Talluri. 2015. «Supply chain risk management: A literature review». International Journal of Production Research 53 (16): 5031-69. https://doi.org/10.1080/00207543.2015.1 030467. 
Hosseini, Seyedmohsen, Dmitry Ivanov, y Alexandre Dolgui. 2019. «Review of quantitative methods for supply chain resilience analysis». Transportation Research Part E: Logistics and Transportation Review 125 (December 2018): 285-307. https://doi.org/10.1016/j. tre.2019.03.001.

Ivanov, Dmitry, y Boris Sokolov. 2010. Adaptive supply chain management [electronic resource]. Editado por Boris Sokolov. 1st ed.. London: London.

Jamalnia, A, J.-B. Yang, A Feili, D.-L. Xu, y G Jamali. 2019. «Aggregate production planning under uncertainty: a comprehensive literature survey and future research directions». International Journal of Advanced Manufacturing Technology 102 (1-4): 159-81. https://doi. org/10.1007/s00170-018-3151-y.

Kazemi, Nima, Nikunja Mohan Modak, y Kannan Govindan. 2018. «A review of reverse logistics and closed loop supply chain management studies published in IJPR: a bibliometric and content analysis». International Journal of Production Research 7543: 1-24. https://doi. org/10.1080/00207543.2018.1471244.

Koberg, Esteban, y Annachiara Longoni. 2019. «A systematic review of sustainable supply chain management in global supply chains». Journal of Cleaner Production 207: 1084-98. https://doi.org/10.1016/j.jclepro.2018.10.033.

Kochan, Cigdem Gonul, y David R Nowicki. 2018. «Supply chain resilience: a systematic literature review and typological framework». International Journal of Physical Distribution \& Logistics Management 48 (8): 842-65. https://doi.org/10.1108/IJPDLM-02-2017-0099.

Malviya, Rakesh Kumar, y Ravi Kant. 2015. «Green supply chain management (GSCM): a structured literature review and research implications». Benchmarking: An International Journal 22 (7): 1360-94. https://doi. org/10.1108/BIJ-01-2014-0001.

Mula, Josefa, David Peidro, Manuel Díaz-Madroñero, y Eduardo Vicens. 2010. «Mathematical programming models for supply chain production and transport planning». European Journal of Operational Research 204 (3): 377-90. https://doi.org/10.1016/j.ejor.2009.09.008.

Mundi, Isabel, M. M. E. Alemany, Raúl Poler, y Vicente S. Fuertes-Miquel. 2019. «Review of mathematical models for production planning under uncertainty due to lack of homogeneity: proposal of a conceptual model». International Journal of Production Research 7543: 1-45. https://doi.org/10.1080/00207543.2019.1566665.
Oliveira, J. B., M. Jin, R. S. Lima, J. E. Kobza, y J. A.B. Montevechi. 2019. «The role of simulation and optimization methods in supply chain risk management: Performance and review standpoints». Simulation Modelling Practice and Theory 92 (June 2018): 17-44. https:// doi.org/10.1016/j.simpat.2018.11.007.

Patel, Ashish Bhanabhai, y Tushar N. Desai. 2018. «A systematic review and meta-analysis of recent developments in sustainable supply chain management». International Journal of Logistics Research and Applications 0 (0): 1-22. https://doi.org/10.1080/13675567.2018.153 4946.

Peidro, David, Josefa Mula, Raúl Poler, y Francisco Cruz Lario. 2009. "Quantitative models for supply chain planning under uncertainty». International Journal of Advanced Manufacturing Technology 43 (3-4): 400420. https://doi.org/10.1007/s00170-008-1715-y.

Pires Ribeiro, João, y Ana Barbosa-Povoa. 2018. «Supply Chain Resilience: Definitions and quantitative modelling approaches - A literature review». Computers and Industrial Engineering 115 (November 2017): 109-22. https://doi.org/10.1016/j.cie.2017.11.006.

Sacaluga, Ana Mejías, Jesús García Arca, J. Carlos Prado Prado, Arturo J.Fernández González, y José A.Comesaña Benavides. 2011. «Modelo para la aplicación de la Responsabilidad Social Corporativa en la Gestión de la Cadena de Suministro». Direccion y Organizacion 45: 20-31.

Sel, Çağrı, y Bilge Bilgen. 2015. «Quantitative models for supply chain management within dairy industry: a review and discussion». European J. of Industrial Engineering 9 (5): 561. https://doi.org/10.1504/ejie.2015.071772.

Seuring, Stefan, y Stefan Gold. 2012. «Conducting content-analysis based literature reviews in supply chain management». Supply Chain Management 17 (5): 54455. https://doi.org/10.1108/13598541211258609.

Seuring, Stefan, y Martin Müller. 2008. «From a literature review to a conceptual framework for sustainable supply chain management». Journal of Cleaner Production 16 (15): 1699-1710. https://doi.org/10.1016/j.jclepro.2008.04.020.

Sodhi, Manmohan S, y Christopher S Tang. 2012. Managing supply chain risk. Vol. 172. International series in operations research \&amp; and management science 172. New York, NY: Springer. 
Soto-Silva, Wladimir E., Esteve Nadal-Roig, Marcela C. González-Araya, y Lluis M. Pla-Aragones. 2016. «Operational research models applied to the fresh fruit supply chain». European Journal of Operational Research 251 (2): 345-55. https://doi.org/10.1016/j.ejor.2015.08.046.

Srivastava, Samir K. 2007. «Green supply-chain management: A state-of-the-art literature review». International Journal of Management Reviews 9 (1): 53-80. https:// doi.org/10.1111/j.1468-2370.2007.00202.x.

Stindt, Dennis, y Ramin Sahamie. 2014. «Review of research on closed loop supply chain management in the process industry». Flexible Services and Manufacturing Journal 26 (1-2): 268-93. https://doi.org/10.1007/ s10696-012-9137-4.
Tseng, Ming Lang, Md Shamimul Islam, Noorliza Karia, Firdaus Ahmad Fauzi, y Samina Afrin. 2019. «A literature review on green supply chain management: Trends and future challenges». Resources, Conservation and Recycling 141 (June 2018): 145-62. https://doi.org/10.1016/j. resconrec.2018.10.009.

Zandi Atashbar, Nasim, Nacima Labadie, y Christian Prins. 2018. «Modelling and optimisation of biomass supply chains: a review». International Journal of Production Research 56 (10): 3482-3506. https://doi.org/10.1080/0 0207543.2017 .1343506 . 